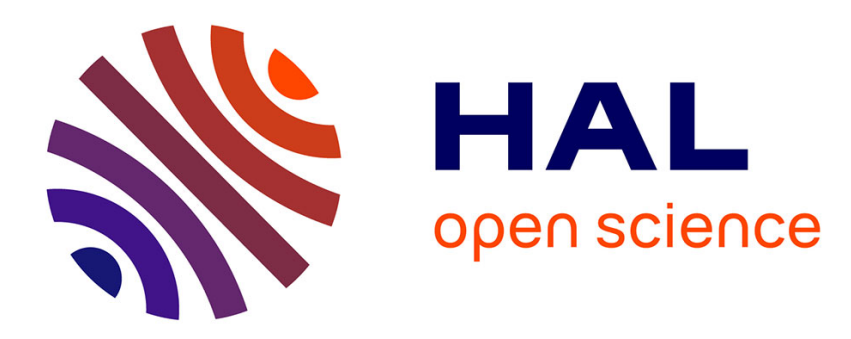

\title{
La pointe nord de Sumatra et la côte orientale de l'Inde : horizons économiques (XIIe s.-XVIIe s.)
}

\author{
Daniel Perret
}

\section{To cite this version:}

Daniel Perret. La pointe nord de Sumatra et la côte orientale de l'Inde: horizons économiques (XIIe s.-XVIIe s.). Archipel, 2014, 87 (1), pp.143-172. 10.3406/arch.2014.4460 . hal-02565692

\section{HAL Id: hal-02565692 \\ https://hal.science/hal-02565692}

Submitted on 6 May 2020

HAL is a multi-disciplinary open access archive for the deposit and dissemination of scientific research documents, whether they are published or not. The documents may come from teaching and research institutions in France or abroad, or from public or private research centers.
L'archive ouverte pluridisciplinaire HAL, est destinée au dépôt et à la diffusion de documents scientifiques de niveau recherche, publiés ou non, émanant des établissements d'enseignement et de recherche français ou étrangers, des laboratoires publics ou privés. 


\section{La pointe nord de Sumatra et la côte orientale de l'Inde :} horizons économiques (XIle s.-XVIle s.)

\section{Daniel Perret}

\section{Citer ce document / Cite this document :}

Perret Daniel. La pointe nord de Sumatra et la côte orientale de I'Inde : horizons économiques (XIle s.-XVIle s.). In: Archipel, volume 87, 2014. Regards croisés sur Aceh. pp. 143-172;

doi : https://doi.org/10.3406/arch.2014.4460

https://www.persee.fr/doc/arch_0044-8613_2014_num_87_1_4460

Fichier pdf généré le 08/11/2019 


\begin{abstract}
The northern Tip of Sumatra and the eastern Coast of India : economic opportunities (12th-17th centuries).

This article considers the economic history of the northern tip of Sumatra in the longue duree by focusing on its interactions with the east coast of India, defined here as the coastline between Chittagong and Cape Comorin, from the twelfth to the seventeenth centuries. It is based on two assumptions : these interactions were continuous during the period under study here, and detailed contemporaneous archaeological data from neighbouring areas, especially from Barus on the west coast, are relevant to remedy the paucity of data available for the northern tip itself. Indian inscriptions and Western sources, whether travelogues or trading companies archives, especially from the VOC, complete the corpus of sources. Three aspects are considered : the bases of the economic positioning of the northern tip of Sumatra in relation to the eastern coast of India, the various categories of actors involved in this economic history, and finally the main trading places on the eastern coast India for which economic contacts with the northern tip of Sumatra were likely or attested during the period under study here.
\end{abstract}

\title{
Résumé
}

Cet article considère l'histoire économique de la pointe nord de Sumatra sur la longue durée en s'intéressant aux rapports qu'elle a entretenus avec la côte orientale de l'Inde, comprise ici entre Chittagong et le cap Comorin, du XIle au XVIle siècles. II se fonde sur deux postulats : ces rapports ont été continus durant la période qui nous intéresse ici et des données archéologiques contemporaines précises accumulées dans des régions voisines, en particulier à Barus, sur la côte ouest, permettent de pallier la rareté des données disponibles pour la pointe nord elle-même. Inscriptions d'origine indienne et témoignages occidentaux, qu'il s'agisse de récits de voyage ou d'archives de compagnies de commerce, en particulier de la VOC, complètent le corpus des sources. Trois aspects sont examinés : les bases du positionnement économique de la pointe nord de Sumatra par rapport à la côte orientale de l'Inde, les différentes catégories d'acteurs de cette histoire économique, et enfin les principales places marchandes de la côte orientale de l'Inde à propos desquelles des contacts économiques avec la pointe nord de Sumatra sont probables ou avérés au cours de la période concernée. 


\section{La pointe nord de Sumatra et la côte orientale de l'Inde : horizons économiques (XII ${ }^{\mathrm{e}} \mathrm{s} .-\mathrm{XVII}{ }^{\mathrm{e}} \mathrm{s}$.)}

Pour la période qui nous intéresse ici, l'historiographie de la pointe nord de Sumatra, que nous limitons arbitrairement à l'actuel territoire administratif d'Aceh, est caractérisée par une division en trois grands volets : un premier ensemble comprend les travaux s'intéressant à tout ce qui peut être relié au toponyme (ou à un ensemble de toponymes associés à) Lambri ou Lamuri. Il peut se définir comme le volet consacré à l'histoire indianisée de la pointe nord de Sumatra, histoire qui prendrait fin symboliquement lorsque Lambri est annexé par Aceh. Le second volet est centré sur l'histoire du sultanat de Pasai, entre la fin du XIII e siècle et 1523, année de sa conquête par Aceh. Le troisième volet recouvre les deux premiers siècles du sultanat d'Aceh.

L'histoire économique de la région s'organise par rapport à cette division. Celle de Lambri est appréhendée à travers des témoignages archéologiques et épigraphiques, ainsi qu'à travers un petit corpus de sources écrites étrangères. A ce jour, il en ressort une connaissance très schématique, qui reflète l'intégration de la région dans des réseaux marchands asiatiques ${ }^{1}$.

1. Il n'est pas question de reprendre ici toutes les données relatives à ce toponyme, ou au groupe de toponymes concernés, dont on suit la trace dans les sources étrangères dès le IX ${ }^{\mathrm{e}}$ siècle EC. Entre le début du XII ${ }^{\mathrm{e}}$ siècle et le début du XVe siècle, diverses sources occidentales et chinoises attestent du rôle de Lambri en tant que place marchande internationale (cf. Kévonian 1998 : 41; Hirth \& Rockhill 1966 : 72, note 1 p. 73 ; Rockhill TP 15(3) 1914 : 439, 440, 442; Rockhill TP 16(1) 1915:148; Polo 1955: 246-7; Pordenone in Yule \& Cordier 1913, II : 148; Ma Huan 1970 : 122-124). 
L'histoire économique de Pasai peut se diviser en deux phases, division non pas fondée sur une rupture dans le déroulement de cette histoire, mais sur la nature et le volume de sources permettant de l'étudier : une première phase qui va jusqu'au milieu du $\mathrm{XV}^{\mathrm{e}}$ siècle, pour laquelle l'état des connaissances est similaire à celui de Lambri; une seconde phase marquée par la relative abondance de sources portugaises qui permettent d'éclairer divers aspects de l'histoire économique des dernières décennies du sultanat de Pasai. C'est sans surprise qu'à ce jour, c'est l'histoire économique du troisième volet qui a suscité le plus d'intérêt de la part des historiens, ceci en raison de la multiplication et de la précision des sources écrites étrangères de première main sur la situation dans la capitale même du sultanat d'Aceh.

Notre idée de départ ici est simple. Plutôt que d'envisager l'histoire économique de la pointe nord de Sumatra selon le découpage traditionnel, nous proposons de la considérer sur la longue durée en nous focalisant sur les rapports qu'elle a entretenus avec la côte orientale de l'Inde, comprise dans cette étude entre Chittagong et le cap Comorin. Il nous semble logique de penser que dans cet espace géographique ces rapports ont été continus durant la période qui nous intéresse ici ${ }^{2}$. Au-delà de l'alternance de phases

Si aucune donnée archéologique ou épigraphique disponible ne permet d'associer avec certitude un site de la pointe nord de Sumatra à Lamuri, Edwards McKinnon qui est pratiquement le seul à avoir conduit des prospections dans ce but, propose plusieurs candidats, tout en précisant l'absence de traces archéologiques de contacts externes pour la période antérieure au XIII ${ }^{\mathrm{e}}$ siècle. Les indices de contacts internationaux apparaissent notamment sous la forme de tessons de céramiques chinoises et de poteries très similaires aux poteries médiévales communes d'Inde et de Sri Lanka. Dans sa publication la plus récente sur la question, il propose Lhok Cut à Ujung Batee Kapal, sur la rive orientale de la baie de Krueng Raya, provisoirement daté entre le XIII e et le XV ${ }^{e}$ siècle, comme le Ramni des sources arabes et l'Ilamuridesam dans l'inscription de Thanjavur (Edwards McKinnon 2011 : $140,144,148)$. Mais plusieurs autres sites anciens ont été repérés à la pointe nord : Neusu dans les faubourgs actuels de Banda Aceh; Lambadeuk et Kuala Pancu dans la baie de Lambaro, un peu à l'ouest de Banda Aceh; Lamreh, Ladong, Cut Me, dans la baie de Krueng Raya, quelque 50 kilomètres à l'est de Banda Aceh (Edwards McKinnon 1988: 118-9; $2003: 163 ; 2006: 327,329,333 ; 2006 b ; 2011: 140)$.

L'apparente stabilité toponymique sur plusieurs siècles masque vraisemblablement une réalité plus complexe, avec des déplacements de centre de gravité, à l'image d'Aru, une entité politique mentionnée sur la côte nord-est de Sumatra entre la fin du XIII ${ }^{\mathrm{e}}$ siècle et le début du XVIII ${ }^{\mathrm{e}}$ siècle. Rappelons aussi le cas du sultanat de Johor, en péninsule malaise, dont la capitale se déplace à vingt reprises entre le milieu des années 1530 et 1718 (Perret \& Kamarudin 1999 : 173). Par ailleurs, les variantes toponymiques contemporaines pourraient indiquer des «chefferies» ou des «maisons» concurrentes, comme à Dar al-Kamal/Makuta Alam de part et d'autre de la rivière Aceh, à Barus/Fansur sur la côte ouest (Perret 2009 : 536-543), ou encore Aru/Deli dans la région actuelle de Medan. Cette configuration n'est pas propre au nord de Sumatra, puisque lorsque Pigafetta est à Brunei en 1521, il mentionne l'existence de deux villes avec chacune son propre roi : l'une est musulmane et reçoit les membres de l'expédition, tandis que l'autre est toujours «païenne» (Stanley (éd.) $1963: 114-6$ ).

2. Deux témoignages contemporains rendent compte de la perception de l'éloignement en termes de journées de navigation. Ainsi, vers 1320, Odoric de Pordenone se rend 


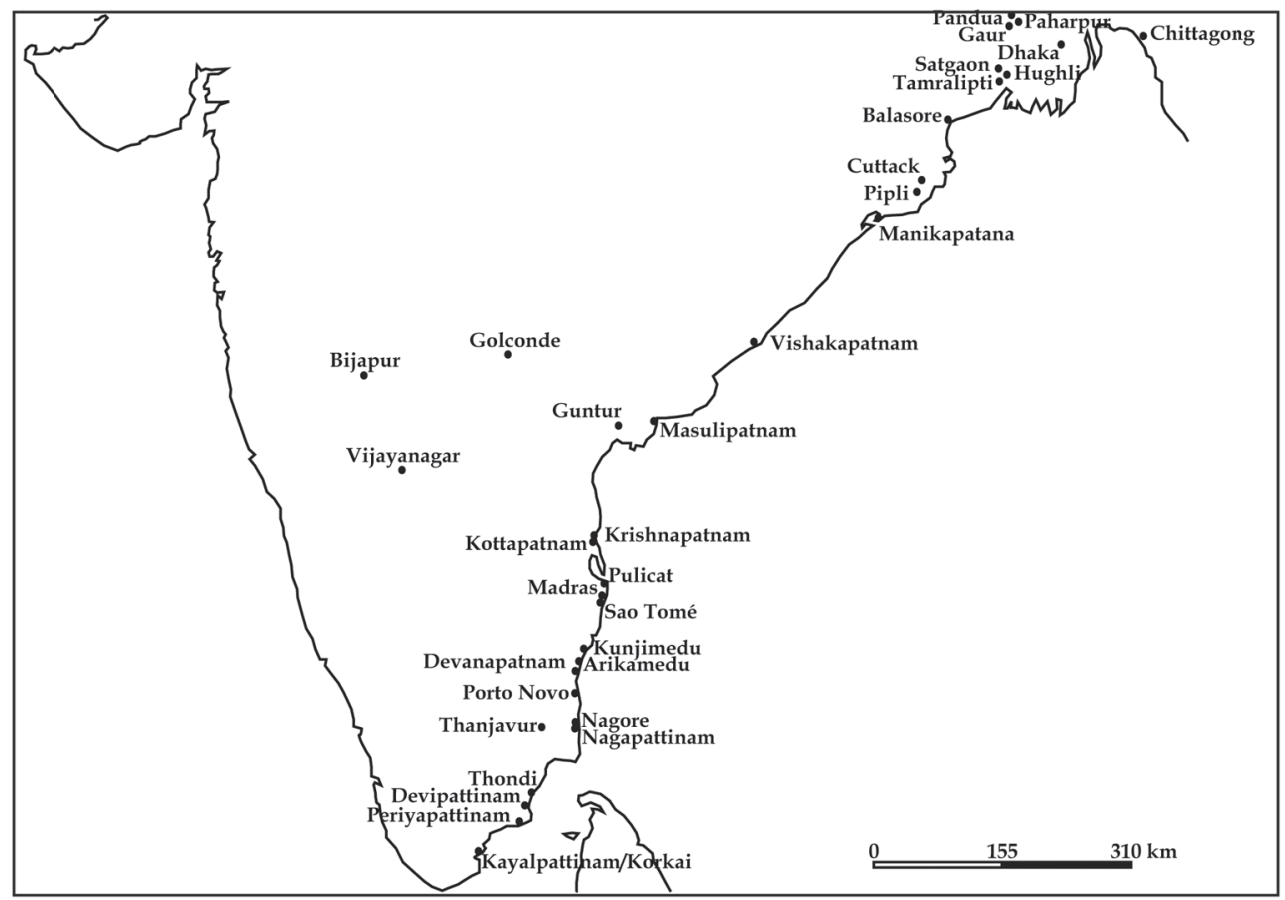

Côte orientale de l'Inde. Principaux toponymes cités 
de déclin et de prospérité, des fluctuations de centres de gravité à l'intérieur de cette zone, évolutions plus ou moins liées à des événements extérieurs ${ }^{3}$, nous supposons que le déterminisme géographique et des différences de potentiels ont généré des attractions économiques d'intensité variable dans les deux sens.

Le caractère fragmentaire des sources empêche de prétendre livrer un panorama exhaustif de ces relations économiques. Tout d'abord, le territoire actuel d'Aceh attend encore ses premières fouilles archéologiques intensives. Les données archéologiques, elles-mêmes limitées dans leur pouvoir explicatif des relations économiques, se cantonnent pour l'instant à des résultats de prospections et de quelques sondages limités. C'est pourquoi nous utiliserons ici des données archéologiques contemporaines précises accumulées dans des régions voisines, en particulier à Barus, sur la côte ouest. Notre postulat est ici que ce qui est valable un peu plus au sud à un moment donné devrait l'être à la même époque pour la pointe nord de Sumatra. Seules des fouilles intensives permettront de valider ces propositions. Il convient cependant de garder à l'esprit que les importants bouleversements côtiers liés à des phénomènes tectoniques ou d'érosion au cours du dernier millénaire ont probablement conduit à la disparition définitive de sites d'habitat contemporains de la période qui nous intéresse ici.

Une seconde raison, qui interdit de prétendre à l'exhaustivité, est que l'activité économique conduite par les marchands de la pointe nord de Sumatra en direction de la côte orientale de l'Inde est très pauvrement documentée. Rappelons ici ce qui a déjà été maintes fois déploré par les historiens, à savoir l'absence quasi totale de références au grand commerce dans les sources écrites locales, que ce soit dans les chroniques anciennes, l'Hikayat Raja Pasai ou l'Hikayat Aceh, pour ce qui concerne la pointe nord $^{4}$, ou dans l'épigraphie islamique, qui est pourtant la plus abondante de l'archipel pour cette période. Un aspect certainement important de cette histoire économique échappe par conséquent à l'investigation.

En dehors des données archéologiques mentionnées ci-dessus, nos sources sont donc des inscriptions d'origine indienne et des témoignages occidentaux, qu'il s'agisse de récits de voyage ou d'archives de compagnies de commerce, en particulier de la VOC.

du Coromandel à Lamuri en 15 jours (in Yule \& Cordier (éd.) 1913, II : 146), alors qu'une vingtaine d'années plus tard, Ibn Baț̣uța met 40 jours pour rallier Pasai, en partant de la côte du Bengale (1995 : 964).

3. Citons par exemple la prise de Melaka par les Portugais en 1511, la prise de contrôle du Bengale par la dynastie moghole ainsi que la mise en place du système de concessions par les Portugais vers 1570, ou encore les conflits entre Aceh et la VOC entre 1640 et 1659.

4. Tout au plus retrouve-t-on à quelques reprises la marque conventionnelle dans les textes malais anciens d'une phase de prospérité indiquée brièvement par l'arrivée de nombreux marchands. 
Trois aspects de l'histoire de ces rapports économiques sont examinés : les bases du positionnement économique de la pointe nord de Sumatra par rapport à la côte orientale de l'Inde, les différentes catégories d'acteurs de cette histoire économique, et enfin les principales places marchandes de la côte orientale de l'Inde à propos desquelles des contacts économiques avec la pointe nord de Sumatra sont probables ou avérés au cours de la période qui nous intéresse ici.

\section{Le positionnement économique de la pointe nord de Sumatra}

Par rapport à la côte orientale de l'Inde, le potentiel économique de la pointe nord de Sumatra se décline en plusieurs volets. Le premier, qui revient le plus souvent dans les études, concerne l'existence de marchés d'échanges de denrées et produits. Au-delà de ses propres richesses (or, pierres semi-précieuses ? ${ }^{5}$, ambre gris ? ${ }^{6}$, corail ?, soufre? ; produits forestiers - camphre ${ }^{7}$, ivoire et éléphants ${ }^{8}$, bois et résines de senteur ${ }^{9}$, bois à colorant ${ }^{10}$, bois de laque ? ${ }^{11}$, rotin, résines à calfatage et autres types de

5. Un témoignage tardif, puisqu'il date de la fin du XIX ${ }^{\mathrm{e}}$ siècle, mentionne l'extraction de rubis à plusieurs journées de marche dans l'intérieur d'Aceh, sur la côte ouest (Saint-Pol-Lias 1892 : 21). Les points d'interrogation dans la liste signalent qu'à notre connaissance, l'exportation vers la côte orientale de l'Inde n'est pas attestée, mais possible.

6. Concrétion biliaire du cachalot (Physeter macrocephalus). La côte nord-ouest de Sumatra est réputée dès la première moitié du $\mathrm{XV}^{\mathrm{e}}$ siècle au moins pour sa richesse en ambre gris, et l'on sait qu'il s'agit d'une denrée exportée de Pasai (Eredia 1930 : 171; Wheatley 1959 : 125-130; Ptak (ed.) $1996: 60-1$ ).

7. Le camphre figure par exemple parmi les principales importations du Bengale, et ce peutêtre dès le XII ${ }^{\mathrm{e}}$ siècle (Ejaz Hussain $2003: 287-288$ ).

8. On sait ainsi que les marchands musulmans du Coromandel et du Bengale fréquentant Aceh aux XVI'-XVII ${ }^{e}$ siècles s'y procurent en particulier de l'ivoire et des éléphants (Boxer 1985 : 427; Lancaster 1940 : 90 (1602); Davis in Purchas 1905, II : 315, 322 (1599); 'Kort verhael ofte journael van de reyse gedaen naar de Oost-Indien... Onder den Admirael Pieter Both...' in Commelin (éd.) 1646, I : 2 (1600); Martin in Pyrard 1998, II : 918; 'Historische Verhael Vande Reyse gedaen inde Oost-Indien....Wybrandt van Waerwijck als Admirael...' in Commelin (éd.) 1646, I : 16 (1603); Jonge 1864-73, III : 153; 'Verhoeven' in Commelin (éd.), 1646, II : 38; Danvers (éd.) 1896, I : 254; Colenbrander \& Coolhaas (éd.) 1919 : 60; 'P. van den Broecke' in Commelin (éd.) 1646, II : 87; Broecke 1648 : 91 (1602); Mundy 1919, vol. III, part I : 135 (1637); Coolhaas (éd.), A. van Diemen et al., Batavia, 22/12/1643, GM II : 217; Tiele et Heeres 1895, vol. 3 : 480 (1649); Arasaratnam 1987 : 100; Manguin 1993 : 203; Stephen 1998:149-150; Subrahmanyam 1990a : 130, 135; Subrahmanyam $1999: 175$.

9. Au XVI ${ }^{\mathrm{e}}$ siècle, les Marakkayar du Coromandel s'approvisionnent en bois d'aigle à Aceh (Stephen 1998 : 149-150; Subrahmanyam 1990a : 130, 135; Subrahmanyam 1999 : 175).

10. Ainsi, les marchands musulmans du Coromandel se fournissent en bois de sapan à Aceh au XVI e siècle (Stephen 1998 : 149-150; Subrahmanyam 1990a : 130, 135; Subrahmanyam 1999 : 175).

11. Utilisé comme encens, il est issu d'une grande liane, Dalbergia parviflora Roxb. Au début du $X^{e}$ siècle, Ma Huan signale que Lambri en fournit la meilleure qualité (1970: 123). 
résines, grumes ou planches de diverses essences, civettes ${ }^{12}$ et musc ${ }^{13}$, cire d'abeille, miel, plantes médicinales ${ }^{14}$-; épices locales - poivre, puwar pelaga ? $^{15}$ pendarah ${ }^{16}$-; produits artisanaux - soie ${ }^{17}$, belacan (pâte de crevette) ? - ), la pointe nord semble offrir très tôt un large éventail des richesses de l'archipel ${ }^{18}$, y compris notamment le benjoin ${ }^{19}$, le clou de girofle $^{20}$ et la noix de muscade des Moluques, l'étain de la péninsule

12. Les civettes vivantes semblent faire partie des importations indiennes, puisqu'un édit du Maharaja Ganapatideva destiné à assurer la sécurité des marchands étrangers venant par la mer (Mottupalli, 1244), les mentionne avec le bois de santal, le camphre, le camphre chinois et l'huile de camphre (Epigraphia Indica, XII, 1913-4, Konow, S. (ed.), Calcutta, Gvt Printing, s.d. : 189). Une civette de Sumatra est bien connue pour fournir du musc, à la fois pour les parfums et en pharmacopée, il s'agit de Viverra tangalunga (en malais : musang tenggalung). Il faut mentionner également une autre espèce de civette de Sumatra, dont le nom pourrait avoir un lien avec Lamuri, puisqu'il s'agit de musang lamri (Paguma larvata). Sa rareté de nos jours pourrait s'expliquer par des captures massives à époque ancienne.

13. C'est un produit importé en Inde puisqu'il figure dans l'inscription de Chintapalle en Andhra Pradesh (1240), au même titre que le camphre (Abraham 1988 : 147). Nikitin (1470) mentionne le musc comme un produit disponible à Shabat, identifié avec la pointe nord de Sumatra (Braginsky 1998 : 378) et, peu après, Ibn Majid rapporte que Sumatra est, entre autres, le pays du musc, dont le prix égale son poids en or (Tibbetts 1979 : 211). On sait qu'aux XVI ${ }^{e}-X V I I^{e}$ siècles, le musc est un produit exporté par Aceh (Iskandar (éd.) 1958 : 164).

14. On note par exemple que la noix d'arec apparaît associée au camphre dans l'inscription tamoule de Kovilpatti (XIII ${ }^{\mathrm{e}}$ siècle) à propos de dons de produits faits par des marchands (Karashima 2002:8). L'intérêt des Indiens pour la noix d'arec de Sumatra est confirmé par Forrest dans la seconde moitié du XVIII ${ }^{\mathrm{e}}$ siècle, qui signale qu'elle est acquise à Aceh par les Chulia venus du Coromandel (1792: 42). Au début du XIX ${ }^{\mathrm{e}}$ siècle, elle est encore exportée d'Aceh vers le Coromandel (Anderson $1840: 163$ ).

15. Selon Braginsky, le texte arménien du XII ${ }^{\mathrm{e}}$ siècle mentionnerait en effet la culture et l'exportation de cardamome à Krut près de Lamuri (1998 : 369). Il est peu probable qu'il s'agisse de la vraie cardamome, la cardamome du Malabar, mais plutôt des fruits séchés d'Amomum compactum Soland. ex Maton (kapulaga à Java; puwar pelaga à Sumatra) (Guzman \& Siemonsma (éd.) 1999 : 68-71).

16. Une graine aromatique utilisée comme la noix de muscade, indigène à Sumatra et absente en Asie du Sud, il s'agit de Myristica cinnamomea King (pendarah en Malaisie) (Guzman \& Siemonsma (éd.) 1999 : 258).

17. Au moins dès le $X V I^{e}$ siècle, la soie de Pasai est exportée vers l'Inde (Salmon 2005 : 244). Beaulieu, qui séjourne au nord de Sumatra en 1621, précise qu'elle est produite en quantité à Pedir, puis acheminée vers Aceh, où une partie est exportée au Coromandel (1996 : 197-8).

18. L'itinéraire arménien du début du XII ${ }^{\mathrm{e}}$ siècle décrit ainsi Lambrē : «Il en sort du brésil en quantité, et il en sort bien d'autres marchandises de prix, on trouve dans ce port tout ce que l'on pourrait désirer» (Kévonian 1998: 41).

19. Dans la seconde moitié du XVIII ${ }^{\mathrm{e}}$ siècle, Marsden exclut Aceh de la zone de production de benjoin $(1986: 154)$. Signalons cependant que la présence d'arbres à benjoin dans les forêts voisines de la capitale d'Aceh est suggérée dans le Hikayat Aceh, un texte daté du début du XVII siècle (Iskandar (éd.) 1958 : 164).

20. A la fin du XIII ${ }^{\mathrm{e}}$ siècle, Marco Polo signale l'abondance de Lambri en clou de girofle (1955: 246). 
malaise, ainsi que des céramiques produites en Chine. Pour des marchands venant d'Asie du Sud, des prix certainement plus élevés par rapport à ceux pratiqués dans la partie orientale de l'archipel devaient être largement compensés par la réduction des risques du voyage maritime, ainsi que par une marge bénéficiaire toujours conséquente.

En sens inverse, la pointe nord de Sumatra importe principalement des cotonnades du Coromandel et du Bengale, ceci probablement dès le début de la période qui nous intéresse $\mathrm{ici}^{21}$, des soieries du Bengale, de l'indigo du Coromandel $^{22}$, du fer et de l'acier du Coromandel ${ }^{23}$, de l'opium $^{24} \mathrm{du}$ Bengale, riz et blé du Bengale, du sel ${ }^{25}$, du beurre et de l'huile du Coromandel et du Bengale, du sucre du Bengale ${ }^{26}$, du sticklack du Bengale, des chevaux ${ }^{27}$, des diamants de Golconde ${ }^{28}$, sans oublier de la maind'œuvre' 29 .

21. Les inscriptions relatives à la guilde marchande Ayyāvole de la fin du XIII ${ }^{\mathrm{e}}$ siècle donnent l'impression d'un accroissement considérable des exportations de cotonnades à destination de l'Asie du Sud-Est (Abraham 1988 : 143-150). Au XVI' siècle, des Marakkayar livrent à Aceh des cotonnades du Coromandel (Subrahmanyam 1990a : 130, 135; Stephen 1998 : 149-150). Caspar Schmalkalden, à Aceh en 1647, y note la popularité des cotonnades apportées de Coromandel et du Bengale (Salmon 2005 : 246).

22. Une importation signalée à Aceh dès le XVI e siècle (Manguin 1993 : 203; Subrahmanyam 1990a : 130,135). Mais les guildes marchandes d'Inde du Sud exportent des produits de teinture à destination de l'Asie du Sud-Est dès le XIII ${ }^{\mathrm{e}}$ siècle au moins (Abraham $1988: 144)$.

23. Le Coromandel fournit Aceh au XVI e siècle (Subrahmanyam 1990a : 130, 135), mais comme les produits de teinture, les guildes marchandes d'Inde du Sud en exportent vers l'Asie du Sud-Est dès le XIII' siècle au moins (Abraham $1988: 171$ ).

24. Lombard $1967: 116$.

25. Forrest précise que le sel est employé comme ballast dans les navires du Coromandel qui se rendent à Aceh au XVIII' siècle (Forrest $1792: 40$ ). Le sel est en fait déjà mentionné dans les inscriptions tamoules comme une denrée négociée par les guildes (Abraham 1988 : 228).

26. Ceci dès le XVI ${ }^{\mathrm{e}}$ siècle (Subrahmanyam 1990a : 130, 135).

27. Le cheval est attesté dans les vestiges de faune de Bukit Hasang, à Barus, probablement dès le XIVe siècle (Rokus 2009). Un trafic important de chevaux est signalé en Asie du Sud aux XII ${ }^{\mathrm{e}}$-XIII ${ }^{\mathrm{e}}$ siècles. Une inscription de la guilde Ayyāvole trouvée au large du nord de Sri Lanka, et datée 1178, fait ainsi référence au commerce de chevaux (Abraham 1988 : 169170). Le Ma'bar (sud Coromandel), avec Kayal et Periyapattinam (Karashima 2002b : 167; Polo 1955 : 274), ainsi que l'Andhra (Abraham 1988: 170) ont été des régions d'importation de chevaux aux XIII $-\mathrm{XIV}^{\mathrm{e}}$ siècles. Ces animaux étaient très probablement acheminés du Moyen-Orient (Aden, Dhufar, Qalhat, Hormuz) (Polo 1955 : 296, 299, 300; Ibn Bațūța $1995: 609)$.

28. Lombard $1967: 116$.

29. Ayant abordé cet aspect dans une précédente publication (cf. Perret $2011: 161-169$ ), nous nous contenterons de rappeler ici qu'à Aceh, cette main-d'oeuvre est employée dans l'agriculture (plantations de poivriers, rizières), la pêche, les activités minières (or), en tant que forces spéciales du palais et gestionnaires de ses domaines, ou encore assiste les gens aisés dans diverses tâches. 
Comme nous l'avons suggéré ailleurs pour Barus ${ }^{30}$, il est probable que des places marchandes de la pointe nord de Sumatra étaient intégrées dans un commerce triangulaire effectué par des marchands installés sur la côte orientale de l'Inde, dans lequel ces derniers apportaient des cargaisons de textiles à la pointe nord, textiles échangés contre des produits locaux, qu'ils acheminaient ensuite vers des ports de Java pour les troquer contre des épices des Moluques, épices ensuite négociés avec grand profit dans les ports indiens.

On sait, grâce à l'inscription tamoule de Kulottunga (1078) de Guangzhou, que le réseau marchand tamoul se prolongeait jusqu'en Chine dès le $\mathrm{XI}^{\mathrm{e}}$ siècle au moins, et s'y est maintenu jusqu'à la fin du XIII siècle ${ }^{31}$. Il est donc tout à fait envisageable que la pointe nord ait aussi joué le rôle de carrefour d'échanges entre deux sections des réseaux marchands indiens, d'une part une section reliant la pointe nord de Sumatra et la côte orientale de l'Inde, d'autre part une section reliant la Chine et la pointe nord de Sumatra. Sachant que la Chine s'alimentait en textiles du Bengale via Pasai ${ }^{32}$, on peut suggérer que la communauté marchande tamoule de Quanzhou assurait une partie de ce trafic, livrant en sens inverse des produits chinois, comme des grès et porcelaines que l'on retrouve sur des sites de la côte indienne ${ }^{33}$. Un réseau marchand chinois pourrait avoir poursuivi ce rôle de relais à la pointe nord de Sumatra dès le $\mathrm{XV}^{\mathrm{e}}$ siècle ${ }^{34}$.

Le second volet concerne l'exploitation directe des ressources naturelles de la pointe nord. L'inscription tamoule de Neusu (fin du XIII siècle), dans les faubourgs de l'actuel Banda Aceh, mentionne des activités liées à l'or de la part de membres d'une guilde ${ }^{35}$. L'exploitation, par des gens venus d'Asie du Sud, de gisements aurifères à la pointe nord de Sumatra, est par conséquent plausible dès cette époque. Cette hypothèse trouverait un écho dans une chronique locale, le Hikayat Raja Pasai, à travers un épisode relatant l'arrivée d'un navire de marchands du pays Keling, sur lequel se

30. Perret 2009 : 626.

31. Cf. l'inscription sino-tamoule (1281) et les vestiges de Quanzhou, attestant de la présence d'une importante communauté tamoule à l'époque (Ray 1995:49; Christie 1998b : 265-266; Karashima 2002a : 15-16; Lee 2009).

32 Ray 1995 : 46.

33. L'existence de relations diplomatiques entre Pasai et la cour de Chine à l'époque est peutêtre liée au rôle de relais joué par Pasai, d'autant que les missions chinoises font ensuite route vers l'Inde. Sur ces relations, voir Guillot \& Kalus $2008: 121$.

34. Le Ming Shi-lu nous apprend ainsi qu'un certain Song Yun, Chinois adjoint de l'envoyé du Bengale, arrive à la cour de Chine en 1439. La même source précise qu'il a un neveu nommé Ai Yan qui habite Samudra en 1446, attestant ainsi d'une implantation chinoise à Pasai au milieu du XV e siècle, en relation avec des Chinois installés au Bengale (Wade 1994, vol. I(2) : 320-1; vol. V(1) : 1222).

35. Subbarayalu 2009b. 
trouve un homme doué du pouvoir de vision de la fumée d'or (asap emas) ${ }^{36}$. Prétendant avoir repéré sept gisements inconnus des autochtones, le souverain lui remet des vêtements coutumiers et l'envoie chercher les sites, d'où il ramène une grande quantité d'or ${ }^{37}$.

Le troisième volet concerne le développement d'activités artisanales par des gens venus de la côte orientale de l'Inde, activités dont les profits seraient réinvestis dans les réseaux marchands indiens ou dont une partie de la production serait exportée vers l'Inde. L'artisanat de la soie pourrait en être l'un des aspects. D'après Pires, au début du XVI ${ }^{\mathrm{e}}$ siècle, la soie est exportée par plusieurs places marchandes de la pointe nord : Daya, Pedir, Lide, Pirada et Pasai ${ }^{38}$. Mais des activités liées à la soie dans la région sont bien antérieures, puisque le texte arménien du début du XII ${ }^{\mathrm{e}}$ siècle mentionne une grande quantité de vers à soie à Lamrin, qui n'est autre que Lamuri ${ }^{39}$. Nous avons développé ailleurs 1'idée que le Bengale a probablement joué un rôle décisif dans le développement de cet artisanat dans la région ${ }^{40}$ et noté plus haut qu'une partie de la production locale de soie est exportée vers le Coromandel dès le XVI ${ }^{e}$ siècle (cf. note 17). Nettement plus spéculative, l'idée qu'en raison de la forte demande du marché local en textiles indiens jusqu'au milieu du XVII ${ }^{\mathrm{e}}$ siècle, notamment ceux du Coromandel et du Bengale pour ce qui nous concerne ici, des entrepreneurs de ces régions auraient développé des plantations de cotonniers et installé des ateliers de production à la pointe nord de Sumatra, un exemple de délocalisation avant la lettre.

\section{Les acteurs du grand commerce maritime}

Les acteurs des échanges économiques entre la pointe nord de Sumatra et la côte orientale de l'Inde appartiennent à des catégories sociales très diverses.

Il y a tout d'abord ceux qu'on pourrait qualifier d'opportunistes. C'est le cas de capitaines de navires qui, dans une même expédition commerciale, peuvent être à la fois marchands indépendants, agents de l'armateur du navire qu'ils pilotent ou encore commissionnaires d'autres marchands. D'autres catégories sociales non marchandes, tels les marins, qui disposent d'espaces pour de petites cargaisons vendues à l'arrivée, se retrouvent également souvent en situation marchande dans la région. Par ailleurs, sur un

36. Hill 1960 : 62, transcrit par 'asfa' et non 'asap'. Selon lui, c'est le mot arabe pour 'filon' - note 56 p. 182.

37. Jones (éd.) $1999: 21$.

38. 1990, I : 140-1, 143, 160-1, 163.

39. Braginsky $1998: 369$.

40. Perret 2009 : 611-612. 
même marché un acteur peut intervenir simultanément avec des statuts différents, par exemple en tant que facteur et en tant que marchand indépendant.

Les acteurs autonomes, c'est-à-dire maîtres du choix des cargaisons et libres de négocier, qui font eux-mêmes le déplacement ou résident à Sumatra, offrent un large spectre de conditions sociales, qui dépend de leur capacité à mobiliser des capitaux : du colporteur muni d'une ou deux balles de textiles financées sur fonds propres au marchand indépendant propriétaire de son navire. Ces individus présentent une grande variété d'affiliations ethniques et religieuses. Les colporteurs, très probablement uniquement des hommes, principalement négociants en textiles, s'embarquent au Coromandel et au Bengale et sont certainement actifs durant toute la période examinée ici. Les navires utilisés ne sont pas uniquement des navires d'Asie du Sud. Il peut s'agir de bateaux locaux ou encore de navires européens. Ainsi, dans les années 1670, des marchands chulia naviguent vers Aceh avec des lettres de navigation hollandaises et anglaises. Ils utilisent aussi le drapeau anglais pour contourner les blocus hollandais ${ }^{41}$.

La seconde catégorie comprend des acteurs à l'autonomie plus ou moins limitée qui opèrent dans le cadre de guildes marchandes, de réseaux familiaux ou religieux, de partenariats avec des acteurs locaux, ou encore dans le cadre de compagnies de commerce occidentales. Une inscription en telugu de Vishakhapatnam, datée de la première décennie du XIII ${ }^{\mathrm{e}}$ siècle, mentionne un marchand de Pāśay membre de la guilde Añjuvaṇnam, un toponyme qui rappelle bien sûr Pasai ${ }^{42}$. Ce texte, ainsi que deux autres inscriptions provenant du même lieu et datées de $1090 \mathrm{EC}$, suggèrent que les membres de cette guilde, marchands d'Asie occidentale majoritairement musulmans à compter du $\mathrm{XI}^{\mathrm{e}}$ siècle, étaient en relation avec la guilde Ayyāvoḷe et même traités en tant que membres de cette autre guilde ${ }^{43}$. Une autre inscription, également datée de la première décennie du XIII ${ }^{\mathrm{e}}$ siècle, retrouvée cette fois près de Nagapattinam, mentionne un autre marchand de Pāśay ayant fait une offrande à un temple local. C'est justement une inscription associée à la guilde Ayyāvoḷe, datée de la fin du XIII ${ }^{\mathrm{e}}$ siècle, qui a été retrouvée à Neusu, dans les faubourgs de l'actuel Banda Aceh ${ }^{44}$. A la

\footnotetext{
41. Arasaratnam 1989: 16 .
}

42. L'ancienneté de ce toponyme dans le nord de Sumatra reste toutefois à préciser. En effet, dans le routier arménien du début du XII ${ }^{\mathrm{e}}$ siècle, le toponyme qui semble être localisé à Pasai, précisemment à l'emplacement de l'actuelle Lhokseumawe, est Samwi (Kévonian 1998 : 83-87), que l'on peut raisonnablement identifier avec le Simaui du début du XVII ${ }^{\mathrm{e}}$ siècle, placé au même endroit par Eredia (1930 : 213). Un rapprochement linguistique entre Samwi et Pasai nous paraît difficile à admettre.

43. Subbarayalu 2009: 163-6.

44. Subbarayalu $2009 b$. 
pointe nord de Sumatra, les implantations de guildes, possible pour Añjuvaṇṇam et certaine pour Ayyāvole, ont pu jouer le rôle de relais dans les échanges avec Java notamment et plus loin avec la Chine.

Les marchands peuvent être également organisés en réseaux de parenté à grande échelle pour la gestion commerciale des places lointaines avec des parents effectuant des rotations de plus ou moins longue durée à des points stratégiques des routes commerciales. En fait, de véritables syndicats familiaux de Chetti et surtout de Chulia, couvrant toute l'Asie du Sud-Est avec, dans la seconde moitié du XVII siècle, une concentration à Aceh notamment, fonctionnent en combinant les positions de marchands itinérants et de facteurs 45 .

A la même époque, des Anglais et des Danois appartenant à des compagnies de commerce occidentales, font notamment commerce d'esclaves entre le Coromandel et Aceh ${ }^{46}$.

La troisième catégorie comprend les agents. Il s'agit principalement de nakhoda et de facteurs. Les capitaines de navires ou nakhoda interviennent en tant qu'agent lorsque le «grand marchand», généralement propriétaire d'un ou plusieurs navires, réside en Asie du Sud. Véritable roi à bord, selon l'expression des lois maritimes, il est alors chargé de gérer la cargaison tout en étant assisté également par des agents ou facteurs installés de manière permanente ou semi-permanente en Asie du Sud-Est maritime, employés qui sont parfois des membres de la famille du "grand marchand». Ces nakhoda disposant d'une double expertise de capitaine et de négociant sont nombreux au Coromandel et vont jouer un rôle croissant dans le commerce au XVII ${ }^{\mathrm{e}}$ siècle ${ }^{47}$. Il convient d'ajouter ici le cas particulier du Bengale entre le $\mathrm{XV}^{\mathrm{e}}$ et le XVII ${ }^{\mathrm{e}}$ siècles, où le nakhoda n'est pas un agent mais fait partie des puissants notables-entrepreneurs de la cour, connus sous le nom générique de ghair mahalli ${ }^{48}$. Au XV $\mathrm{V}^{\mathrm{e}}$ siècle, ils font du commerce avec Pasai et Pedir ${ }^{49}$, et au milieu du XVII ${ }^{\mathrm{e}}$ siècle, un prince du Bengale, en l'occurence peut-être un ghair mahalli, passe à Aceh afin de présenter la totalité de sa cargaison à la reine en échange d'éléphants ${ }^{50}$. Parmi les quelques noms de ces agents parvenus jusqu'à nous, il convient de citer Haji Mahmet, agent de Mir Shah Deli, le gouverneur de Pulicat. Dès la fin des années 1650 au moins, on le

45. Arasaratnam 1986 : 215 ; Arasaratnam 1994a : 41, 144; Arasaratnam 1995c : 127, 135.

46. Martineau 1932 : 493-4. Voir aussi Dampier $1715: 3$.

47. Arasaratnam 1994a : 181-182.

48. Subrahmanyam $1999: 63,65$.

49. Ibid. : 63.

50. Andaya $2001: 88$. Sur le commerce des éléphants à Aceh à cette époque, voir notamment Ito (1994). 
voit transporter à Aceh une cargaison de son maître comprenant des textiles, du fer et de l'acier ${ }^{51}$.

Les "grands marchands» en question appartiennent généralement au secteur privé. Les sources occidentales du XVII siècle nous livrent quelques noms. Chinnana, un Telugu implanté en Pays tamoul, est membre de la communauté marchande balija chetti du Coromandel. Grand armateur, ses bases sont Pulicat et Devanampatnam. L'un de ses frères, Kesava Chetti, est également armateur. Basé à Puducheri, on repère sa trace en 1639 au moment où il envoie des navires vers Aceh ${ }^{52}$. Mir Kamaluddin de Masulipatnam ${ }^{53}$ et Astrappah Chetty de Pulicat/Devanampatnam possèdent chacun de cinq à dix navires ${ }^{54}$. Citons également Nella Tamby Sitty de Porto Novo ${ }^{55}$.

Mais ces «grands marchands» peuvent être également des officiels, dirigeants aussi bien que hauts fonctionnaires ou hauts responsables militaires. À la fin du XVI ${ }^{\mathrm{e}}$ siècle, c'est le gouverneur du Bengale lui-même, Sa'id Khan Chaghatai, qui envoie ses navires de commerce à Aceh ${ }^{56}$. Au XVII ${ }^{\mathrm{e}}$ siècle, des membres de la famille impériale, des subahdar et dewan de provinces, des faudjar et syahbandar de ports ou encore des commandants de districts militaires de l'empire moghol participent au grand commerce maritime, actifs à la fois dans la construction navale et en tant qu'armateurs. Ainsi, au Bengale, le commerce avec l'Asie du Sud-Est maritime, en particulier avec Aceh, est complétement dominé par des gens comme le prince Shah Shuja, vice-roi (subahdar) entre 1639 et 1660; son successeur Nawab Mir Muhammad Said, l'ancien Mir Jumla de Golconde passé chez les Moghols; le nawab de Dhaka, Nawab Shaista Khan; Faudjar Ahmed Beg et le syahbandar d'Hughli, le syahbandar de Balesore, le syahbandar de Pipli, ou encore Faudjar Nawazish Khan de Rajmahal, à l'époque une cité sur les rives du Gange, aujourd'hui dans 1'Etat de Jharkhand. En Orissa, il faut mentionner le nawab de Cuttack, Nawab Inoriya Muhammad, ainsi que Diwan Malik Beg 57.

\section{Andaya $2001: 96$.}

52. Il semble posséder une dizaine de navires qui fréquentent divers ports de la baie du Bengale (Subrahmanyam 1990b : 309, 310, 313).

53. Des Persans ou Indo-Persans de Masulipatnam font partie de ces armateurs importants du Coromandel, au moins dès le milieu du XVII ${ }^{\mathrm{e}}$ siècle (Arasaratnam $1991: 40$ ).

54. Arasaratnam 1994a : 179. Durant la seconde moitié du XVII ${ }^{\mathrm{e}}$ siècle, les flottes des plus grands armateurs privés du Coromandel ne dépassent pas dix navires (Arasaratnam 1991 : 39).

55. Chijs et al. (éd.), DR 21/12/1680: 823. Durant la seconde moitié du XVII' siècle, Porto Novo, ainsi que Cuddalore et Nagore, sont les bases de quelques grands armateurs chulia (Arasaratnam 1991: 40).

56. Alam \& Subrahmanyam $2005: 228-229$.

57. Arasaratnam 1994a : 154, 156, 215 ; Subrahmanyam 1999 : 72, 74; Prakash 2004a : 197198; Prakash 2004b : 451. 
En ce qui concerne Golconde, les acteurs les plus importants sont Mir Kamaluddin ainsi que Mir Muhammad Said, le Mir Jumla du sultanat, figure dominante du transport maritime du Coromandel durant deux décennies. Dans la seconde moitié du XVII ${ }^{\mathrm{e}}$ siècle, les principaux acteurs du trafic d'esclaves entre le Coromandel et Aceh sont à la fois de hauts personnages du Coromandel, tels Nawab Mosemchan (ou Chanchanan), ainsi que le souverain de Golconde ${ }^{58}$. Dans les années 1680, les personnages de premier plan dans le transport maritime du Coromandel sont Mir Abdullah Baguir et Mir Fakiruddin 59.

Ce «grand marchand» n'est pas toujours un Asiatique. Ainsi en 1602, Lancaster rencontre à Aceh un marchand indien au service d'un capitaine portugais, venu du Bengale avec un navire chargé de riz. Cet Indien explique qu'il sert le capitaine portugais, mais n'est ni lié ni libre. Il a été avec ce capitaine depuis si longtemps que ce capitaine le considère maintenant presque comme l'un des siens ${ }^{60}$.

À moins d'un contexte particulier de fermeture au grand commerce, l'élite dirigeante locale a bien sûr tout intérêt à attirer un maximum de marchands, ceux-ci étant non seulement synonymes de taxes et autres prélévements, mais aussi de revenu pour l'élite en général. Perçus comme des opportunités d'obtention de capitaux financiers, ils fournissent l'accès à des marchés mondiaux à l'ouest tout en introduisant des biens de prestige et de valeur prisés par les dirigeants. De plus, ces étrangers les assistent grâce à leur expertise dans l'administration du commerce. L'une des stratégies des dirigeants locaux est de jouer sur les taxes pour fidéliser tel ou tel groupe. Ainsi, dans les réglements portuaires d'Aceh du XVII ${ }^{\mathrm{e}}$ siècle ${ }^{61}$, une distinction est faite à propos du tarif du droit d'accostage (cap) entre les navires du Gujerat, du Kalinga, du Bengale et d'Europe qui paient deux fois plus cher que les navires du Coromandel, du Malabar, des Maldives, de Pegu et de péninsule malaise ${ }^{62}$. Une autre stratégie consiste à accorder des tarifs préférentiels sur telle ou telle denrée ou marchandise. Ainsi, en 1621, le souverain d'Aceh autorise la vente du poivre à un tarif préférentiel aux marchands de Masulipatnam, tarif inférieur à celui appliqué aux Hollandais ${ }^{63}$.

58. Ito $1984: 400$.

59. Arasaratnam 1994b : 25 .

60. Lancaster $1940: 90$.

61. La place prépondérante faite aux navires et aux marchands indiens dans ces réglements traduit le rôle essentiel des Indiens dans le commerce d'Aceh, probablement dès les premières décennies du sultanat (Ito $1984: 305$ ).

62. Ibid. : 311 . Les droits d'importation sont par contre pratiquement équivalents (Ito 1996 : 38).

63. Raychaudhuri $1962: 120$. 
Pour l'élite impliquée directement dans le grand commerce, en général par la possession d'un ou plusieurs navires, le partenariat avec des marchands d'Asie du Sud ouvre non seulement la possibilité d'écouler ses cargaisons dans certains ports en bénéficiant de leur savoir-faire, de leur expérience et de leur capital social, mais aussi la perspective d'accès à des financements extérieurs qui sur le plan intérieur renforcent probablement son prestige et l'avantagent par rapport aux rivaux politiques. Cette conjonction d'intérêts se traduit généralement par la mise en place de partenariats et d'agences dans les cours locales. Ainsi, au début du XVII siècle, le sultanat de Golconde, qui a des agents dans toute l'Asie du Sud-Est, dispose de relais à Aceh, terminal majeur dans le commerce du Coromandel à l'époque. C'est peut-être dans le cadre d'un partenariat avec Golconde qu'Iskandar Muda envoie chaque année un grand navire au Coromandel ${ }^{64}$.

Cette initiative d'Iskandar Muda soulève la question du rôle des acteurs de la pointe nord de Sumatra même dans les relations économiques avec la côte orientale. S'agit-il d'autochtones ou d'individus originaires d'Inde plus ou moins intégrés dans les sociétés locales? Font-ils partie des équipages faisant la navette à l'intérieur de l'espace considéré ? Forment-ils de véritables communautés installées dans les ports indiens? Les données manquent malheureusement pour répondre à ces questions.

A l'inverse, l'activité des communautés marchandes de la côte orientale de l'Inde en direction de la pointe nord de Sumatra étant relativement bien documentée, il est clair que des membres de ces communautés étaient installés en particulier à Pasai ${ }^{65}$, Pedir ${ }^{66}$ et Aceh ${ }^{67}$. D'après certaines traces archéologiques et l'inscription de Neusu, il devait en être de même dans les ports plus anciens de la pointe nord. Sans doute dès le début de la période qui nous intéresse ici, les acteurs sont non seulement des autochtones (Keling, Chetti, Marakkayar, Labbai, Chulia ${ }^{68}$ ), mais aussi des individus originaires de la côte occidentale (gens du Malabar, du Konkan, du Gujerat), ainsi que des Persans, Arabes, Turcs, Afghans, Pathans, Abyssiniens, Arméniens ${ }^{69}$, sans oublier les Chinois mentionnés plus haut.

64. Reid 1993, II : 31, 250.

65. On trouve des allusions à cette présence dans le Hikayat Raja Pasai. Cf. également Pires 1990, I : 143-144; II : 240; Reid 1993, II : 120; Alves 1994 : 135; Alves 1999 : 119-123; Alves 2001; Guillot \& Kalus 2008 : 116-119.

66. Situé à l'ouest de Pasai, ce port était en contact économique avec la côte orientale de l'Inde dès le début du XVI ${ }^{\mathrm{e}}$ siècle au moins. Pires mentionne ainsi le passage annuel de deux navires du Bengale, ainsi que d'un navire du Coromandel (1990, I : 139). Voir également Noonan 1989: 192; Alves 2003 : tableau au verso de p. 176.

67. Roy, ca. 1700 : 126; "Davis" in Purchas 1905, II : 315; Lombard 1967 : 47; Alves 1990 : 102; Alves \& Manguin (éd.) 1997 : 71; Manguin 2011: 237.

68. Arasaratnam 1989 : 18; Arasaratnam 1995c : 128; Arasaratnam $1996: 162-163$.

69. Meilink-Roelofsz 1962 : 68; Bouchon 1987 : 166, 174; Subrahmanyam 1990a : 22, 119; 
Il convient d'ajouter les Danois à cette liste. En raison de leurs liens anciens et étroits avec les Chulia, ils fournissent la collaboration la plus significative, prenant du frêt et des passagers, parfois plusieurs centaines de marchands, dans leurs quatre grands bateaux faisant le trajet, accordant des lettres de navigation et même des pavillons à de nombreux bateaux chulia en route pour des ports non contrôlés par la VOC, y compris Aceh. Avec les Anglais, ils semblent avoir été tout particulièrement actifs dans le commerce des esclaves entre le Coromandel et Aceh durant la seconde moitié du XVII ${ }^{\mathrm{e}}$ siècle ${ }^{70}$.

\section{Les places marchandes de la côte orientale de l'Inde}

La combinaison des données relatives au mobilier archéologique probablement originaire d'Asie du Sud collecté sur le territoire d'Aceh et à Barus avec des données archéologiques et épigraphiques d'Asie du Sud même, ainsi que des informations livrées par des sources étrangères contemporaines, nous permet de suggérer une liste des principaux centres de la côte orientale de 1'Inde, avec lesquels la pointe nord de Sumatra a pu être en contact entre le $\mathrm{XII}^{\mathrm{e}}$ et le XVII ${ }^{\mathrm{e}}$ siècles.

En partant du nord, à proximité du delta du Gange, Tamralipti ${ }^{71}$, le plus important port de grand commerce maritime du Bengale jusqu'aux XI ${ }^{\mathrm{e}} / \mathrm{XII}^{\mathrm{e}}$ siècles est à considérer pour le tout début de la période qui nous intéresse ici. En lien avec cette hypothèse, il convient de mentionner que du mobilier religieux de Padang Lawas, à Sumatra Nord, daté entre le $\mathrm{IX}^{\mathrm{e}}$ et les $\mathrm{XI}^{\mathrm{e}} / \mathrm{XII}^{\mathrm{e}}$ siècles, montre des traits qui rappellent ceux communément observés dans le sud-est du Bangladesh actuel (Bautze-Picron, à paraître). Ces rapprochements dans le domaine religieux à propos d'une région voisine de la pointe nord invitent à envisager également l'existence de contacts économiques.

Du matériel très voisin de celui découvert sur le site de Paharpur, au nord-ouest du Bangladesh (district de Birbhum), ayant été identifié sur le site de Barus-Bukit Hasang avec une datation entre le XII et le XVe siècle ${ }^{72}$, des contacts entre Paharpur et la pointe nord de Sumatra à cette époque sont tout à fait envisageables.

Le site de Bukit Hasang, à Barus, a également livré un type de poterie, daté entre le $\mathrm{XIII}^{\mathrm{e}}$ et le $\mathrm{XV}^{\mathrm{e}}$ siècle, qui serait une production de la cité de

Subrahmanyam 1990b : 94, 96, 108; Arasaratnam 1994a : 125; Winks 1996 : 272 ; Subrahmanyam 1997 : 44; Alves 1999 : 75, note 25; Alves 2003 : 28; Prakash 2004b : 451.

70. Martineau 1932 : 493-4. Voir aussi Dampier $1715: 3$.

71. Il est généralement admis aujourd'hui que le site se trouve sur l'emplacement de l'actuelle Tamluk dans le district de Midnapore.

72. Perret et al. 2009 : 235-6, 270. 
Gaur $^{73}$, l'ancienne Lakhnauti. Cette place est par conséquent à considérer comme un contact probable de la pointe nord à l'époque. Dès la fondation du sultanat sous l'autorité de Delhi, Lakhnauti en devient la capitale et restera capitale d'une "principauté» de taille plus réduite jusqu'en 1341. La ville abrite de nombreux étrangers, en particuliers des gens venus d'Asie centrale et de Perse ${ }^{74}$.

Sous le règne de Sikandar Shāh (ca. 1356-ca. 1390), le Bengale, devenu sultanat indépendant, développe des relations amicales avec l'empereur Ming et le grand commerce maritime (avec la Chine, Sumatra, la Perse, le Yémen, etc...) s'opère via Chittagong, ici notre limite orientale sur la côte indienne, alors que la capitale est à Pandua/Firuzabad ${ }^{75}$, ville qui entretient également des contacts commerciaux et culturels avec la Chine notamment ${ }^{76}$.

Dès les années 1570, Aceh devient la tête de pont d'un réseau concurrent de celui de l'Estado da India, comprenant notamment plusieurs ports du Bengale, en particulier Hughli, qui prend le relais de Satgaon. La présence de navires du Bengale à Aceh, en particulier de navires musulmans, est rapportée régulièrement dans les sources occidentales jusqu'à la fin du XVII ${ }^{\mathrm{e}}$ siècle ${ }^{77}$. Nous avons mentionné plus haut qu'au cours de ce siècle des hauts personnages de Dhaka et de Hughli prennent part au commerce avec Aceh.

Un certain nombre de types de poteries du site de Barus-Bukit Hasang, datés des débuts de la période qui nous intéresse ici, montrent des similitudes avec des types identifiés en Orissa ${ }^{78}$. Le port le plus pertinent pour avancer l'idée de contacts avec la pointe nord de Sumatra nous semble être Manikapatana, à $45 \mathrm{~km}$ au sud-ouest de Puri, sur le canal reliant le lac Chilika à la mer, un site où de nombreuses céramiques chinoises des XIIIe$\mathrm{XIV}^{\mathrm{e}}$ siècles ont été collectées ${ }^{79}$, reflétant une probable orientation des

73. Perret et al. $2009: 227,229-230,280$.

74. Ejaz Hussain $2003: 244$.

75. La capitale précédente et postérieure est Lakhnauti/Gaur (1205-1341 et 1434-1572) (ibid. : 242).

76. ibid. : 96, 253, 283.

77. 'Davis' in Purchas 1905, II : 315, 322 (1599); 'Kort verhael ofte journael van de reyse gedaen naar de Oost-Indien... Onder den Admirael Pieter Both...' in Commelin (éd.) 1646, I: 2 (1600); Lancaster 1940 : 90 (année 1602); 'Historische Verhael Vande Reyse gedaen inde Oost-Indien....Wybrandt van Waerwijck als Admirael...' in Commelin (éd.) 1646, I : 16 (1603); 'P. van den Broecke' in Commelin (éd.) 1646, II : 87; Broecke 1648 : 91 (1602); Mundy 1919, vol. III, part I : 135 (1637); Coolhaas (éd.) A. van Diemen et al., Batavia, 22/12/1643, GM II : 217; Tiele et Heeres 1895, vol. 3 : 480 (1649); Coolhaas (éd.), C. Reniers et al., Batavia, 20/01/1651, GM II : 462; Coolhaas (éd.), C. Reniers et al., Batavia, 24/01/1652, GM II : 569; Chijs et al. (éd.), DR 27/01/1661 : 16; DR 5/12/1663 : 634; DR 08/03/1665 : 48 ; Bowrey $1905: 287-8$ (années 1670).

78. Perret et al. $2009: 200-201$.

79. Pradham et al. 2000; Basa \& Behera $2000: 573$; Tripati \& Vora $2005: 1177$. 
activités commerciales vers l'Asie du Sud-Est. Les rapports entre l'Orissa et l'Asie du Sud-Est maritime semblent très peu documentés dans les sources écrites. On sait néanmoins que des marchands de l'Orissa fréquentent Melaka dès la seconde moitié du $\mathrm{XV}^{\mathrm{e}}$ siècle $^{80}$, ouvrant ainsi la possibilité de relations avec Pasai. Des ports de l'Orissa sont en contact avec Aceh dès les années 1560, et nous avons déjà souligné l'implication de hauts personnages de Cuttack, Balasore et Pipli dans le commerce avec Aceh au XVII ${ }^{\mathrm{e}}$ siècle. Des navires de Balasore fréquentent toujours Aceh durant les dernières décennies du siècle ${ }^{81}$.

Nous avons indiqué plus haut la relation probable entre le port de Vishakhapatnam et la pointe nord, à travers une inscription de guilde marchande datée de la première décennie du XIII ${ }^{\mathrm{e}}$ siècle.

Sur la côte du Coromandel, six inscriptions de guildes ont été recensées pour l'instant dans le delta du fleuve Krishna, précisemment dans le district de Guntur. Quatre sont datées entre 1240 et 1269 : Chintapalle (1240), Motupalli (1244), Velpuru (1244) et Enamadala (1269). Les deux premières y mentionnent des produits disponibles à la pointe nord de Sumatra à l'époque, à savoir l'or, le camphre, l'huile de camphre, le musc, les civettes, les éléphants, l'ivoire, et la noix d'arec ${ }^{82}$. Les deux autres textes proviennent de Motupalli à nouveau (1358) et d'Appapuram (1405) ${ }^{83}$.

Les relations entre la pointe nord de Sumatra et le delta du fleuve Krishna ont probablement été relancées dès la fin des années 1560, après la chute de Vijayanagar au profit de Golconde. Golconde va encourager l'immigration non seulement de Persans mais aussi de populations originaires d'autres régions du Moyen-Orient (Afghans, Pathans, Arabes) ${ }^{84}$. Le sultanat attire par ailleurs de nombreux Gujarati qui vont jouer un rôle important dans les activités marchandes de son port de Masulipatnam, débouché maritime de la nouvelle zone de croissance commerciale créée par le sultanat qui dispose d'un arrière-pays très productif ${ }^{85}$. Masulipatnam va immédiatement se positionner en port du Coromandel prédominant dans les échanges avec Aceh et les deux places resteront en contact commerciaux jusqu'au milieu du $\mathrm{XVII}^{\mathrm{e}}$ siècle $^{86}$. Ces relations sont fondées essentiellement sur les réseaux

80. Pires 1990, II : 268; A.K. Das Gupta $1987: 247$; Thomaz $1993: 82$.

81. Prakash $1963: 40-41$.

82. Abraham 1988 : 147; Karashima (éd.) 2002 : 293-4.

83. Karashima (éd.) 2002 : 295-6. Fouillé dans les années 1970, le site de Motupalli est daté XIII'-XIVe siècles (Subbarayalu, comm. pers., avril 2008).

84. Subrahmanyam $1999: 66$.

85. Schrieke 1957, II : 392, note 133; Subrahmanyam 1990b : 113; Subrahmanyam 1997 : $44,57$.

86. Martin in Pyrard 1998, II : 918; 'Historische Verhael Vande Reyse gedaen inde Oost- 
marchands musulmans fonctionnant hors du contrôle portugais, réseaux qui ici semblent dominés par les Gujarati ${ }^{87}$. Cette domination musulmane sur le commerce de Masulipatnam s'accompagne probablement d'un retrait partiel des marchands hindous du nord Coromandel dans le commerce avec le monde insulindien.

Plus au sud, Krishnapatnam, au nord de l'embouchure du fleuve Upputeru, dans le district de Nellore (actuel Andhra Pradesh), a livré deux inscriptions de guildes marchandes (XII ${ }^{\mathrm{e}}$ siècle et 1279$)^{88}$. De très nombreux tessons de céramiques chinoises et thaïes datées XIII -XIV siècles ont été collectés à Kottapatnam ${ }^{89}$, une implantation au sud de l'embouchure du même fleuve. Ces deux sites sont par conséquent des candidats potentiels pour des contacts avec la pointe nord entre le XII et le $\mathrm{XIV}^{\mathrm{e}}$ siècle.

Le port de Pulicat, lié à la cité royale de Vijayanagar, capitale d'un royaume hindou fondé durant la décennie 1340, est probablement en relation avec la pointe nord de Sumatra dès la fin du $\mathrm{XV}^{\mathrm{e}}$ siècle. On sait ainsi que durant les deux premières décennies du $\mathrm{XVI}^{\mathrm{e}}$ siècle, des navires de Pulicat fréquentent Pasai ${ }^{90}$. Pulicat abrite une population marchande mixte en contact avec l'Asie du Sud-Est maritime. Déjà sévèrement touché par le système de concessions (carreira) entre l'Inde et l'archipel insulindien mis en place par l'Estado da India de façon effective à la fin des années 1550, Pulicat connaît un grand déclin après la chute de Vijayanagar en 1565. Néanmoins Aceh reçoit encore des navires de Pulicat durant la première moitié du XVII ${ }^{\mathrm{e}}$ siècle, bien qu'il s'agisse alors d'une petite cité ne comptant guère plus de deux à trois mille habitants ${ }^{91}$.

Nagapattinam a livré une inscription de la guilde Ayyāvoḷe datée 1090 EC, mentionnant qu'un temple y a été construit pour le bien-être des orfèvres ou par des orfèvres ${ }^{92}$. La présence d'orfèvres dans ce port, probablement Kaveripattinam (ca. 900-1200), renvoie bien sûr à l'importation d'une

Indien....Wybrandt van Waerwijck als Admirael...' in Commelin (éd.) 1646, I : 16; Foster (éd.) 1899, III : Nicholls, p. 234; Sen 1962 : 96; Colenbrander \& Coolhaas (éd.) VII(2) : 932; Coolhaas (éd.), Carpentier et al., GM I, 13/12/1626 : 204; 'P. van den Broecke' in Commelin (éd.) 1646, II: 87; Broecke 1648: 91; Tiele et Heeres 1895, vol. 2 : 307-308; Mundy, 1919, vol. III, part I : 135 (1637), part II : 329; Coolhaas (éd.), A. van Diemen et al., Batavia, 22/12/1643, GM II : 217; Subrahmanyam 1990a : 130, 135.

87. Bouchon \& Thomaz (éd.) $1988: 159$.

88. Karashima (éd.) $2002: 302$.

89. Rao 2002; Sasaki 2002.

90. Noonan 1989: 193; Stephen 1998 : 134, 149; Alves 1999: 119; Alves 2001 : 130; Alves 2003 : 141-142, tableau au verso de p. 176.

91. Colenbrander \& Coolhaas (éd.), VII(1) : 398; Arasaratnam 1986 : 119; Stephen 1998 : 207 (1615).

92. Karashima \& Subbarayalu $2002: 59$. 
matière première disponible à la pointe nord de Sumatra. Des contacts avec des sites de la vallée de la Kaveri sont également probables plus tard, puisqu'à l'époque de l'inscription de la guilde marchande Ayyāvoḷe à Neusu, trois inscriptions de guildes datées entre le milieu du XIII ${ }^{\mathrm{e}}$ siècle et le début du XIV ${ }^{\mathrm{e}}$ siècle, retrouvées dans le district de Tiruchirapalli, à une centaine de kilomètres à l'intérieur des terres, à hauteur de Nagapattinam, mentionnent une série de produits disponibles à la pointe nord : camphre, huile de camphre, civettes, bois d'aloès, cire, miel, noix d'arec ${ }^{93}$. Des navires de Nagapattinam fréquentent Pasai dès le début du $\mathrm{XVI}^{\mathrm{e}}$ siècle au moins ${ }^{94}$ et Nagapattinam sera même, avec Masulipatnam, le port prédominant du Coromandel en rapport avec Aceh dans le dernier quart du $\mathrm{XVI}^{\mathrm{e}}$ siècle $^{95}$. Les armateurs de Nagapattinam commercent encore activement avec Aceh dans les années 1630 et $1640^{96}$ et les contacts commerciaux perdurent jusque dans la seconde moitié du XVII ${ }^{\mathrm{e}}$ siècle $^{97}$.

Les Marakkayar basés à Nagore, un port voisin de Nagapattinam, font régulièrement du commerce à Aceh dès sa fondation, à raison de trois bateaux par an. Ils sont souvent associés à des marchands gujerati qui chargent leurs navires à Kollam au Malabar avant de se rendre à Nagore d'où ils font voile vers Aceh avec des marchands du Coromandel ${ }^{98}$.

L'ancien port d'Arikamedu, près de Pondicherry, a connu une phase médiévale datée XIII ${ }^{\mathrm{e}}$ XV ${ }^{\mathrm{e}}$ siècles, apparemment surtout $\mathrm{XIV}^{\mathrm{e}}$ siècle $^{99}$. Du mobilier de ce site ayant pu être rapproché de matériel retrouvé dans les fouilles récentes de Barus ${ }^{100}$, des contacts commerciaux sont par conséquent probables avec la pointe nord.

Durant la première moitié du XVII ${ }^{\mathrm{e}}$ siècle, Aceh reçoit également des navires de Kunjimedu, São Tomé, Devanampatnam et Porto Novo ${ }^{101}$. Le trafic est tel que Coen va jusqu'à affirmer que les marchands musulmans du

93. Abraham $1988: 146$.

94. Pires 1990, I : 143 ; Noonan 1989 : 193; Stephen 1998 : 134, 149; Alves 1999 : 119 ; Alves 2001: 130; Alves $2003: 141-142$.

95. Subrahmanyam 1990a: 130, 135 .

96. Subrahmanyam $1990 \mathrm{~b}: 209$.

97. Coolhaas (éd.), C. Reniers et al., Batavia, 20/01/1651 : 460, GM II : 462; Coolhaas (éd.), Maetsuyker et al., Batavia, 24/12/1655, GM III : 19; Coolhaas (éd.), Maetsuyker et al., Batavia, 4/12/1656, GM III : 93; Andaya 2001 : 96; Chijs et al. (éd.), DR, 22/05/1663 : 212 ; DR 5/12/1663 : 634; DR 08/03/1665 : 48; Bowrey 1905 : 287-8 (années 1670); Arasaratnam 1986 : 149; Subrahmanyam 1990a : 236; Arasaratnam 1995c : 128.

98. Stephen $1998: 149-150$.

99. Begley et al. 2004.

100. Perret et al. 2009.

101. Colenbrander \& Coolhaas (éd.), VII(1) : 398; Arasaratnam 1986 : 119; Stephen 1998 : 207 (1615). 
Coromandel (avec ceux du Konkan - du port de Dabhol en particulier) dominent le commerce extérieur d'Aceh à la fin des années $1620^{102}$. Ce rôle de premier plan du Coromandel se poursuit jusque dans le dernier quart du XVII ${ }^{\text {siècle }}{ }^{103}$, surtout par l'intermédiaire de Porto Novo ${ }^{104}$.

$\mathrm{Au}$ moins dès la dernière décennie du XVII ${ }^{\mathrm{e}}$ siècle, des navires de l'East India Company assurent la liaison Madras-Aceh, transportant notamment des marchands itinérants avec leur frêt et des esclaves ${ }^{105}$.

Dans le détroit de Palk, deux ports du côté indien (Ma'bar) ont pu être en contact avec la pointe nord de Sumatra: Thondi, à l'est de Madurai, dans le district de Ramanathapuram, qui a été actif notamment entre le XII ${ }^{\mathrm{e}}$ et le $\mathrm{XIV}^{\mathrm{e}}$ siècles ${ }^{106}$; Devipattinam, un peu au sud de Thondi, identifié avec le Mali-Fitan (ou Mali-Fatan) des historiens persans du XIV siècle ${ }^{107}$. Une inscription de guilde marchande datée 1270 a d'ailleurs été retrouvée sur ce site ${ }^{108}$.

Entre le XIII ${ }^{\mathrm{e}}$ siècle et le milieu du XIV $\mathrm{X}^{\mathrm{e}}$ siècle, des contacts sont envisageables entre la pointe nord de Sumatra et la région de Kayal, dans le golfe de Mannar, un peu au sud de Tuticorin, avec ses trois ports de PalaiyaKayal (Cail de Marco Polo, Jiayi des sources chinoises), Punnaikayal et Kayalpattinam contrôlés d'abord par le royaume Pandya, puis par le sultanat de Madurai. Une inscription de guilde marchande datée 1282 a été découverte sur le site de Kayalpattinam ${ }^{109}$, qui prendra la suite de PalaiyaKayal victime d'envasement. Dans la même zone, Punnaikayal succédera aussi à Palaiya-Kayal. Marco Polo fait escale à Cail à la fin du XIII ${ }^{\mathrm{e}}$ siècle et mentionne sa fréquentation par de nombreux navires du Moyen-Orient (Hormuz, Qays, péninsule arabique), tout en louant la qualité de son emplacement, le grand nombre de négociants d'origines diverses qui y affluent, ainsi que l'attitude très favorable du souverain à l'égard des marchands étrangers ${ }^{110}$. Kayal, qui a des contacts directs avec la Chine par l'envoi ou la réception d'émissaires à la fin du XIII ${ }^{\mathrm{e}}$ siècle, est encore mentionné par Wang Dayuan en 1349. Une des grandes expéditions Ming passe à Kayal et la cour de Chine reçoit un émissaire de Kayal à plusieurs reprises entre 1411 et 1433 , mais la région ne joue plus un rôle aussi

102. Colenbrander \& Coolhaas (éd.), V : 31.

103. Arasaratnam 1986 : 149; Arasaratnam 1995c : 128; Gommans 1995 : 93.

104. Chijs et al. (éd.), DR 5/12/1663 : 634; Arasaratnam 1986 : 149.

105. Bassett $1989: 630$.

106. Sridhar (éd.) $2004: 38,42$.

107. Karashima $2002 b$ : 168.

108. Karashima (éd.) $2002: 294,299$.

109. Ibid. : 294, 301.

110. Polo 1955 : 274. 
important qu'auparavant ${ }^{111}$. Toujours, dans le golfe de Mannar, il faut citer le port de Periyapattinam, près de Rameswaram, dans le district de Ramnad. Daté XIII ${ }^{\mathrm{e}}-\mathrm{XIV}^{\mathrm{e}}$ siècles, il est identifié par Karashima avec le Fattan d'Ibn Battūṭa (ca. 1345) et le Dabadan dans le Daoyi Zhilue de Wang Dayuan $(1349)^{112}$. À Piranmalai, quelque 80 kilomètres à l'intérieur des terres, dans le même district de Ramnad, une inscription du XIII ${ }^{\mathrm{e}}$ siècle relative à la rénovation et la maintenance d'un temple śivaïte par une communauté marchande itinérante, fait mention de taxes sur certains produits d'échange, dont le bois d'aloès, le camphre et l'huile de camphre ${ }^{113}$.

\section{Conclusion}

Aucun site d'habitat daté du XII' siècle n'ayant été repéré pour l'instant à la pointe nord de Sumatra, c'est ici à partir de matériel identifié dans les fouilles de Barus - Bukit Hasang, du contenu d'inscriptions de guildes marchandes en Inde du Sud et de données archéologiques concernant la côte orientale de l'Inde, que nous avons pu suggérer les noms de plusieurs sites ayant pu être en contact avec la pointe nord de Sumatra à l'époque, qu'il s'agisse de Tamralipti au Bengale, de Krishnapatnam en Andhra Pradesh, ou de sites du delta de la Kaveri au Tamil Nadu. La liste s'allonge à partir du $\mathrm{XIII}^{\mathrm{e}}$ siècle, mais il reste encore beaucoup à faire sur le plan archéologique au nord de Sumatra et sur la côte orientale de l'Inde pour vérifier les hypothèses avancées ici et certainement compléter la carte jusqu'au début du $\mathrm{XV}^{\mathrm{e}}$ siècle.

A ce stade, nous proposons deux zones qui semblent avoir été en contact économique significatif avec la pointe nord de Sumatra durant pratiquement toute la période considérée : le delta du Gange, avec notamment Tamralipti pour le début et Hughli pour la fin du XVII ${ }^{\mathrm{e}}$ siècle; le delta de la Kaveri, avec notamment Kaveripattinam pour le début et Nagapattinam pour la seconde moitié du XVII ${ }^{\mathrm{e}}$ siècle.

En contrepoint à ces continuités, nous décelons plusieurs moments de rupture importants dans l'histoire économique de cet espace. Il y aurait tout d'abord entre la fin du XIII ${ }^{\mathrm{e}}$ siècle et le début du XIV ${ }^{\mathrm{e}}$ siècle la disparition des guildes indiennes à la pointe nord de Sumatra, un phénomène que nous associons à l'émergence d'un nouveau système politique à Pasai. Le centre de gravité politique et économique se déplacerait alors des comptoirs des baies de Lambaro et de Krueng Raya, possiblement dominés par des communautés hindoues, vers Pasai dirigé par des musulmans. Ce moment

111. Ptak 1993.

112. Subbarayalu 1996 : 110; Karashima \& Kanazawa 2002 : 109-111; Karashima 2002.

113. Pathmanathan $2002: 42-4$. 
charnière consacre aussi peut-être la mainmise des réseaux de parenté à grande échelle, des «maisons» marchandes et des associations commerciales ponctuelles sur les relations économiques entre les deux régions.

Nous plaçons un second tournant important vers 1565-1570. Aceh devient la tête de pont d'un réseau musulman concurrent de celui de l'Estado da India, qui intègre plusieurs ports du Coromandel et du Bengale. C'est en même temps la chute de Vijayanagar (1565), qui provoque le déplacement du centre de gravité pour le grand commerce maritime de Pulicat vers Masulipatnam, débouché maritime de Golconde, dont l'élite soutient activement le grand commerce. Cette décennie voit aussi le développement de deux ports importants pour le commerce avec Aceh, surtout Nagapattinam, mais aussi Hughli.

Nous situons le troisième moment de rupture important dans les années 1640. Cette décennie est en effet marquée par l'implication croissante des classes dirigeantes de plusieurs régions d'Inde dans le commerce à destination de l'Asie du Sud-Est maritime. C'est sans doute l'apogée du modèle politico-commercial dans lequel le «grand marchand»s'appuie sur des moyens matériels et des capitaux détenus par l'élite politique et administrative du pays d'origine. Les années 1640 voient aussi le renouveau du grand commerce au Bengale, ainsi que le déclin des industries textiles du Gujerat au profit du Coromandel.

La conjoncture se renverse vers 1660. Aussi bien au Bengale qu'à Golconde, l'élite dirigeante commence à se désengager du grand commerce maritime. Une double tendance expliquerait ce repli général : baisse de rentabilité du commerce avec l'Asie du Sud-Est maritime en raison de la concurrence accrue, forte croissance de la demande européenne en produits indiens qui aurait transformé l'implication de l'élite, abandonnant l'activité d'armateur pour celle beaucoup moins risquée d'exportateur. Le volet transport est, quant à lui, surtout laissé aux compagnies européennes et à leurs associés privés. A la pointe nord, Aceh décline au profit de Johor, au sud de la péninsule malaise.

Ce retrait se confirme avec l'annexion de Golconde et de Bijapur par les Moghols (1686-7). Le nouveau pouvoir se retire des entreprises de transport maritime. Les liens entre monde politique et monde marchand sont clairement rompus, de même que les liens entre ces deux États et leurs anciens «partenaires» d'Asie du Sud-Est maritime. Le même phénomène se produit au Bengale. Parallèlement, les marchands du Coromandel font face à un autre phénomène nouveau : la concurrence croissante des marchands européens privés.

Les dernières décennies de la période étudiée ici donnent l'image d'un abandon progressif de la navigation en direction de l'Asie du Sud-Est maritime par les marchands d'Asie du Sud, à l'exception des Chulia encore 
bien présents dans le détroit de Malacca et à Aceh. Le moindre renouvellement ou l'absence de renouvellement des communautés certainement largement masculines installées dans l'archipel à la fin du XVII siècle conduit à leur évanescence par assimilation plus ou moins rapide.

Tableau récapitulatif des ports de la côte orientale de l'Inde en contacts avérés ou probables avec la pointe nord de Sumatra (XII $-\mathrm{XVII}{ }^{\mathrm{e}}$ siècles)

\begin{tabular}{|c|c|c|c|c|c|c|}
\hline Ports & $\mathrm{XII}^{\mathrm{e}} \mathrm{s}$. & XIIII $^{\mathrm{e}}$ s. & $\mathrm{XIV}^{\mathrm{e}} \mathrm{s}$. & $\mathbf{X V} \mathbf{V}^{\mathrm{e}} \mathbf{s}$ & $X_{V I}{ }^{\mathrm{e}} \mathbf{s .}$ & XVII $^{\mathrm{e}} \mathrm{s}$. \\
\hline Pandua & & & $\mathrm{X}$ & & & \\
\hline Paharpur & $\mathrm{X}$ & $\mathrm{X}$ & $\mathrm{X}$ & $\mathrm{X}$ & & \\
\hline Gaur & & $\mathrm{X}$ & $\mathrm{X}$ & $X$ & & \\
\hline Chittagong & & & $\mathrm{X}$ & & & \\
\hline Dhaka & & & & & & $\mathrm{X}$ \\
\hline Satgaon & & & & & $\mathrm{X}$ & \\
\hline Hughli & & & & & $\mathrm{X}$ & $\mathrm{X}$ \\
\hline Tamralipti & $\mathrm{X}$ & & & & & \\
\hline Balasore & & & & & & $\mathrm{X}$ \\
\hline Cuttack & & & & & & $\mathrm{X}$ \\
\hline Pipli & & & & & & $\mathrm{X}$ \\
\hline Manikapatana & & $\mathrm{X}$ & $\mathrm{X}$ & & & \\
\hline Vishakapatnam & & $\mathrm{X}$ & & & & \\
\hline Masulipatnam & & & & & $\mathrm{X}$ & $\mathrm{X}$ \\
\hline Guntur & & $\mathrm{X}$ & $\mathrm{X}$ & $\mathrm{X}$ & & \\
\hline Krisnapatnam & $\mathrm{X}$ & $\mathrm{X}$ & & & & \\
\hline Kottapatinam & & $X$ & $X$ & & & \\
\hline Pulicat & & & & $\mathrm{X}$ & $\mathrm{X}$ & $\mathrm{X}$ \\
\hline Madras & & & & & & $\mathrm{X}$ \\
\hline Sao Tomé & & & & & & $\mathrm{X}$ \\
\hline Kunjimedu & & & & & & $\mathrm{X}$ \\
\hline Arikamedu & & $\mathrm{X}$ & $\mathrm{X}$ & $\mathrm{X}$ & & \\
\hline Devanapatnam & & & & & & $\mathrm{X}$ \\
\hline Porto Novo & & & & & & $\mathrm{X}$ \\
\hline Nagore & & & & & $\mathrm{X}$ & \\
\hline Nagapattinam & $\mathrm{X}$ & $\mathrm{X}$ & $\mathrm{X}$ & $\mathrm{X}$ & $\mathrm{X}$ & $\mathrm{X}$ \\
\hline Thondi & $\mathrm{X}$ & $\mathrm{X}$ & $\mathrm{X}$ & & & \\
\hline Devipattinam & & $\mathrm{X}$ & $\mathrm{X}$ & & & \\
\hline Periyapattinam & & $\mathrm{X}$ & $\mathrm{X}$ & & & \\
\hline Kayal & & $\mathrm{X}$ & $\mathrm{X}$ & $\mathrm{X}$ & & \\
\hline
\end{tabular}




\title{
RÉFÉRENCES
}

\author{
Abréviations \\ BEFEO \\ Bulletin de l'École française d'Extrême-Orient \\ BKI \\ DR \\ GM \\ IESHR \\ JESHO \\ JEWMR \\ JMBRAS \\ JSEAH \\ MAS \\ PEFEO \\ VKI \\ Bijdragen tot de Taal-, Land- en Volkenkunde van de Koninklijk Instituut \\ Dagh-Register \\ Generale Missive \\ Indian Economic and Social History Review \\ Journal of the Economic and Social History of the Orient \\ Journal of East-West Maritime Relations \\ Journal of the Malayan Branch, Royal Asiatic Society \\ Journal of South-East Asian History \\ Modern Asian Studies \\ Publications de l'Ecole française d'Extrême-Orient \\ Verhandelingen van de Koninklijk Instituut
}

\section{Sources primaires}

Alves, Jorge M. Santos \& Manguin, Pierre-Yves (éd.), O Roteiro das Cousas do Achem de D. João Ribeiro Gaio, Lisboa, Commissão Nacional para as Comemorações dos Descobrimentos Portugueses, 1997.

Anderson, John, Acheen, and the Ports on the North and East Coasts of Sumatra..., London, Wm. H. Allen and Co, 1840.

Beaulieu, Augustin de, Mémoires d'un voyage aux Indes Orientales, 1619-1622. Introduction, notes et bibliographie de Denys Lombard, Paris, École française d'ExtrêmeOrient/Maisonneuve \& Larose, 1996.

Bouchon, Geneviève \& Thomaz, L.P. (ed.), - Voyage dans les deltas du Gange, 1521, Paris, Fondation Calouste Gulbenkian/Centre Culturel Portugais, 1988.

Bowrey, Thomas, A geographical account of countries round the Bay of Bengal, 1669 to 1679, R.C. Temple (ed.), Cambridge, Hakluyt Soc., sec. Series no. xii, 1905.

Broecke, Pieter van den, Wonderlijke Historische ende Journaelische Aenteyckeningh..., Amstelredam, Joost Hartgerts, 1648.

Chijs, J.A. van der et al. (ed.), Daghregister gehouden int Casteel Batavia vant passerende daer ter plaetse als over geheel Nederlandts-India (1624-1682), Batavia, 1887-1928, 21 vol.

Colenbrander, H.T. \& Coolhaas, W. Ph. (ed.), Jan Pietersz Coen: bescheiden omtrent zijn bedrijf in Indie, 's Gravenhage, Martinus Nijhoff, 1919-1953, 7 vol.

Commelin, Is. (ed.), Begin ende voortgangh, van de Vereenighde Nederlantsche Geoctroyeerde Oost-Indische Compagnie..., [Amsterdam, Jan Jansz.], 1646, 2 vol.

Coolhaas, W. Ph. (ed.), Generale Missiven van Gouverneurs-Generaal en Raden aan Heren XVII der Verenigde Oostindische Compagnie (1610-1713), 's-Gravenhage, M. Nijhoff, 1960-1976, 6 vol.

Dampier, Guillaume, Suplement du voyage autour du monde contenant une description d'Achin..., Paris, chez Eustache Herault, 1715.

Danvers, Frederick Charles, Letters received by the East India Company from its servants in the East, 1602-1617, London, Sampson Low \& Co., 1896, vol. I.

Eredia, Godinho de, "Eredia's Description of Malacca, Meridional India, and Cathay. J.V. Mills (trad. \& éd.)", JMBRAS, 8(1), 1930: 1-288.

Forrest, T., A Voyage from Calcutta to the Mergui Archipelago..., London, Robson; Edinburgh, Balfour, 1792. 
Foster, William (ed.), Letters received by the East India Company from its servants...vol. 2-6 (1613-1617), London, Sampson Low, Marston \& Cie, 1897-1902.

Hill, A.H. (ed. \& transl.), "Hikayat Raja-Raja Pasai”, JMBRAS, 33(2), 1960: 1-215.

Hirth, Friedrich \& Rockhill, W.W. (trad. \& éd.), Chau Ju-kua: His Work on the Chinese and Arab Trade in the Twelfth and Thirteenth Centuries, entitled Chu-fan-chï, St. Petersburg, Printing office of the Imperial Academy of Sciences, 1911 (réimpr. Amsterdam, Oriental Press, 1966).

Ibn Batțuța, «Voyages et périples», in P. Charles-Dominique (trad. \& éd.), Voyageurs arabes, Paris, Gallimard, 1995 : 369-1050, 1130-1213.

Iskandar, Teuku (éd.), De Hikajat Atjéh, 's-Gravenhage, Nijhoff (KITLV, VKI 26), 1958.

Jones, Russell (ed.), Hikayat Raja Pasai, Kuala Lumpur, Yayasan Karyawan dan Penerbit Fajar Bakti, 1999.

Jonge, J.K.J. de, De opkomst van het Nederlandsch Gezag in Oost-Indie, 's Gravenhage, M. Nijhoff, 1864-1873, vol. 2-5, 7.

Karashima, Noburu, "South Indian and Sri Lankan Inscriptions Relating to the Merchant Guilds", in N. Karashima (éd.), Ancient and Medieval Commercial Activities in the Indian Ocean: Testimony of Inscriptions and Ceramic-sherds, Taisho Univ., Report on the Taisho Univ. Research Project, 1997-2000, 2002: 3-9.

Lancaster, James, The Voyages of Sir James Lancaster to Brazil and the East Indies, 15911603, W. Foster (éd.), London, Hakluyt Soc., sec. series no. LXXXV, 1940.

Ma Huan, Ying-yai Sheng-lan. The Overall Survey of the Ocean's Shore (1433). Ma Huan, traduit et édité par J.V.G. Mills, Cambridge, Hakluyt Soc. Extra series 42, Cambridge Univ. Press, 1970.

Marsden, William, The History of Sumatra, Singapore, Oxford University Press, 1986 (1 ${ }^{\text {re }}$ éd., Londres, 1783).

Martin, François, «Description du premier voyage fait à Sumatra par les Français en l'an 1603 par François Martin de Vitré», in X. de Castro (éd.), Voyage de Pyrard de Laval, Paris, Chandeigne, 1998, vol. 2 : 905-932 (1 ère éd., Paris, 1603).

Martineau, A. (éd.), Mémoires de François Martin, Fondateur de Pondichéry (1665-1694), Paris, Société de l'histoire des colonies françaises, 1932, vol. 2.

Mundy, Peter, The Travels of Peter Mundy, in Europe and Asia, 1608-1667, R.C. Temple (éd.), London, Hakluyt Soc., 1919, 5 vol.

Noonan, Laurence A., John of Empoli and his relations with Afonso de Albuquerque, Lisboa, Ministério da Educaçāo, Instituti de Investigaçāo Cientifica Tropical, 1989.

Pires, Tomé, The Suma Oriental of Tomé Pires, Armando Cortesão (trad. \& ed.), New Delhi/Madras, Asian Educational Services, 1990, 2 vol. (1 ${ }^{\text {ere }}$ ed., 1944).

Polo, Marco, Marco Polo. La Description du Monde, édité par L. Hambis, Paris, Klincksieck, 1955.

Ptak, Roderich (éd.), Hsing-Ch'a Sheng-Lan. The Overall Survey of the Star Raft by Fei-Hsin, J.V.G. Mills (trad.), Wiesbaden, Harrassowitz Verlag, 1996.

Purchas, Samuel (ed.), Hakluytus posthumus or Purchas his pelgrimes..., Glasgow, MacLehose, vol. II, 1905.

Pyrard de Laval, Voyage de Pyrard de Laval aux Indes orientales (1601-1611). Edité par Xavier de Castro, Paris, Chandeigne, 1998, 2 vol. (1 $1^{\mathrm{ere}}$ ed., 1611).

Rockhill, W.W., "Notes on the Relations and Trade of China with the Eastern Archipelago and the Coast of the Indian Ocean during the Fourteenth Century", T'oung Pao, 15(3), 1914: 419-447; 16(1), 1915: 61-159, 236-271, 374-392, 435-467, 604-626.

Roy, Jacob Janssen de, Voyage Gedaan door J.J. de R. Na Borneo en Atchin In't Jaar 1691 en vervolgens, Amsterdam, [ca. 1700]. 
Saint-Pol-Lias, Brau de, La côte du poivre, Paris, Lecène, Oudin et Cie, 1892.

Stanley, H.E.J.S. (éd.), The first voyage round the world, by Magellan, translated from the accounts of Pigafetta and other contemporary writers, New York, B. Franklin, 1963 (1 ère éd., 1874).

Tibbetts, G.R., A Study of Arabic Texts Containing Material on Southeast Asia, Leiden/London, E.J. Brill, Oriental Translation Fund, new ser. 44, 1979.

Tiele, P.A. \& Heeres, J.E., Bouwstoffen voor de geschiedenis der Nederlanders in den maleischen archipel, 's Gravenhage, M. Nijhoff, 1895, vol. 2-3.

Wade, Geoffroy P., The Ming Shi-lu (Veritable Records of the Ming Dynasty) as a Source for Southeast Asian History: Fourteenth to Seventeenth Centuries, 7 vols., Hong Kong: Univ. of Hong Kong, PhD Dissertation, 1994 (également accessible sur Internet: http://epress.nus.edu.sg/msl/).

Wheatley, Paul, "Geographical Notes on some Commodities involved in Sung Maritime Trade", JMBRAS, 32(2), 1959: 1-139.

Yule, Henry \& Cordier, Henri (trad. \& éd.), Cathay and the Way Thither. Vol. II, London, Hakluyt Society, sec. series, vol. 33, 1913.

\section{Études}

Abraham, Meera, Two Medieval Merchant Guilds of South India, New Delhi, Manohar Publications, 1988.

Alam, Muzaffar \& Subrahmanyam, Sanjay, "Southeast Asia as Seen from Mughal India: Tahir Muhammad's 'Immaculate Garden' (ca. 1600)”, Archipel, 70, 2005: 209-237.

Alves, Jorge M. Santos, «Une Ville inquiète et un Sultan barricadé : Aceh vers 1588 d'après le Roteiro das Cousas do Achem de l'Evêque de Malaka», Archipel, 39, 1990 : 93-112.

—, «Princes contre marchands au crépuscule de Pasai (c. 1494-1521)», Archipel, 47, 1994: 125-146.

-, O Domínio do norte de Samatra, Lisboa, Sociedade Histórica da independência de Portugal, 1999.

-, «Nayinar Kuniyappan. Un Tamoul, syahbandar de Samudera-Pasai au début du XVI ${ }^{\mathrm{e}}$ siècle», Archipel, 62, $2001:$ 127-142.

-, Três Sultanatos malaios do Estreito de Malaca nos séculos XV E XVI (Samudera-Pasai, Aceh E Malaca/Johor), Tesa de Doutoramento, Lisboa, Universidade Nova de Lisboa, 2003.

Andaya, Leonard, "The Seventeenth-Century Acehnese Model of Malay Society", in F. Hüsken \& D. van der Meij (éd.), Reading Asia. New Research in Asian Studies, Richmond, Curzon Press, 2001: 83-109.

Arasaratnam, Sinappah, Merchants, Companies and Commerce on the Coromandel Coast, 1650-1740, Delhi, Oxford University Press, 1986.

-, "Indian and the Indian Ocean in the Seventeenth Century", in A. Das Gupta \& M.N. Pearson (éd.), India and the Indian Ocean, 1500-1800, Calcutta, Oxford University Press, 1987: 94-130.

—, "Islamic Merchant Communities of the Indian Subcontinent in Southeast Asia", Sixth Sri Lanka Endowment Fund Lecture delivered at the University of Malaya, Kuala Lumpur, 1989.

—, "Merchants of Coromandel in Trade and Entrepreneurship circa 1650-1700", in R. Ptak $\&$ D. Rothermund (éd.), Emporia, commodities and entrepreneurs in Asian maritime trade, c. 1400-1750, Stuttgart, Franz Steiner, Beiträge zur Südasienforschung, Bd. 141, 1991: 37-51.

—, Maritime India in the Seventeenth Century, Delhi, Oxford University Press, 1994a. 
—, "Coromandel Shipping and Seafaring in the Indian Ocean, 1650-1800", JEWMR, 3, 1994b: 19-41.

-, "The Chulia Muslim Merchants in Southeast Asia, 1650-1800", in Maritime Trade, Society and European Influence in Southern Asia, 1600-1800, Aldershot, Variorum, 1995c: 125-143 (original publ., MOOI, 1987); in S. Subrahmanyam (ed.), Merchant Networks in the Early Modern World, Aldershot, Variorum, An Expanding World, 1996, vol. 8: 159-177.

Basa, Kishor K. \& Behera, Karuna Sagar, "Maritime Archaeology of Orissa", in Kishor K. Basa \& Pradeep Mohanty (éd.), Archaeology of Orissa, Delhi, Pratibha Prakashan, 2000, vol. II: 566-600.

Bassett, D.K., "British 'Country' Trade and Local Trade Networks in the Thai and Malay States, c. 1680-1770", MAS, 23(4), 1989: 625-643.

Bautze-Picron, Claudine, "Buddhist Images from Padang Lawas Region and the South Asian Connection", in D. Perret (ed.), History of Padang Lawas, North Sumatra. II: Societies of Padang Lawas ( $9^{\text {th }}$ c.-1 $13^{\text {th }}$ c. CE), Paris, Cahiers d'Archipel, forthcoming.

Begley, Vimala; Francis, Peter; Karashima, Noboru; Raman, K.V.; Sidebotham, Steven E.; Lyding Will, Elisabeth, The Ancient Port of Arikamedu. New Excavations and Researches, 1989-1992, vol. two, Paris, École française d'Extrême-Orient, 2004.

Bouchon, Geneviève, "Sixteenth century Malabar and the Indian Ocean", in A. Das Gupta \& M.N. Pearson (éd.), India and the Indian Ocean, 1500-1800, Calcutta, Oxford University Press, 1987: 162-184.

Boxer, C.R., "A note on Portuguese reactions to the revival of the red Sea spice trade and the rise of Atjeh, 1540-1600", in Portuguese Conquest and Commerce in Southern Asian, 1500-1750, Aldershot, Ashgate, Variorum, 1985: 415-428 (1 1 ère éd. JSEAH, X, 1969).

Braginsky, V.Y., “Two Eastern Sources on Medieval Nusantara”, BKI, 54(3), 1998: 367-396.

Das Gupta, Arun Kumar, "The Maritime Trade of Indonesia: 1500-1800”, in A. Das Gupta \& M.N. Pearson (éd.), India and the Indian Ocean, 1500-1800, Calcutta, Oxford University Press, 1987: 240-275.

Edwards McKinnon, E., "Historic Period Earthenware from the Island of Sumatra", in J.N. Miksic (ed.), Earthenware in Southeast Asia, Singapore, Singapore University Press, 2003: 162-172.

-, "Mediaeval Landfall Sites in Aceh, North Sumatra", in E.A. Bacus; I.C. Glover \& V. Pigott (ed.), Uncovering Southeast Asia's past, Singapore, Singapore University Press, 2006: 325-334.

-, "Indian and Indonesian Elements in Early North Sumatra", in A. Reid (ed.), Verandah of violence: The background to the Aceh problem, Seattle, Univ. of Washington Press, 2006b: 22-37.

-, "Continuity and Change in South Indian Involvment in Northern Sumatra: The Inferences of Archaeological Evidence from Kota Cina and Lamreh", in P.-Y. Manguin, A. Mani, G. Wade (eds.), Early Interactions between South and Southeast Asia. Reflections on CrossCultural Exchange, Singapore, ISEAS, India, Manohar, 2011: 137-160.

Ejaz Hussain, Syed, The Bengal Sultanate. Politics, Economy and Coins (AD 1205-1576), Delhi, Manohar, 2003.

Gommans, Jos, "Trade and Civilization around the Bay of Bengal, c. 1650-1800", Itinerario, 19(3), 1995: 82-108.

Guillot, Claude \& Kalus, Ludvik, Les monuments funéraires et l'histoire du sultanat de Pasai à Sumatra (XIII ${ }^{e}-X V I^{e}$ siècles), Paris, Cahier d'Archipel 37, 2008.

Guzman, C.C. de \& Siemonsma, J.S. (éd.), Plant Resources of South-East Asia. No 13: Spices, Bogor, PROSEA, 1999.

Ito, Takeshi, The World of the Adat Aceh, $\mathrm{PhD}$, Canberra, Australian National University, 1984. 
-, "Trade and taxation in Seventeenth Century Aceh", JEWMR, 4, 1996: 35-60.

Karashima, Noboru, "Periyapattinam: An Important Medieval Port on the Coromandel Coast and Its Identification", in N. Karashima (éd.), Ancient and Medieval Commercial Activities in the Indian Ocean: Testimony of Inscriptions and Ceramic-sherds, Taisho Univ., Report on the Taisho Univ. Research Project, 1997-2000, 2002b: 164-169.

Karashima, Noboru (éd.), Ancient and Medieval Commercial Activities in the Indian Ocean: Testimony of Inscriptions and Ceramic-sherds, Taisho Univ., Report on the Taisho Univ. Research Project, 1997-2000, 2002.

Karashima, N. \& Kanazawa, Y., "Testimony of Chinese Ceramic Sherds", in N. Karashima (éd.), Ancient and Medieval Commercial Activities in the Indian Ocean: Testimony of Inscriptions and Ceramic-sherds, Taisho Univ., Report on the Taisho Univ. Research Project, 1997-2000, 2002: 109-118.

—, "Goldsmiths and Padinen-vishayam: A Bronze Buddha Image of Nagapattinam", in N. Karashima (éd.), Ancient and Medieval Commercial Activities in the Indian Ocean: Testimony of Inscriptions and Ceramic-sherds, Taisho Univ., Report on the Taisho Univ. Research Project, 1997-2000, 2002: 57-61.

Kevonian, Kéram, «Un itinéraire arménien de la mer de Chine», in C. Guillot (éd.), Histoire de Barus, Sumatra. Le site de Lobu Tua. I : Etudes et Documents, Paris, Cahier d'Archipel 30, $1998: 35-118$.

Lee, Risha, "Rethinking Community: The Indic Carvings of Quanzhou", in H. Kulke, K. Kesavapany, V. Sakhuja (eds.), Nagapattinam to Suvarnadwipa: Reflections on the Chola Naval Expeditions to Southeast Asia, Singapore, ISEAS, 2009: 240-270.

Lombard, Denys, Le sultanat d'Atjéh au temps d'Iskandar Muda : 1607-1636, Paris, École française d'Extrême-Orient, PEFEO 61, 1967.

Manguin, Pierre Yves, "The Vanishing Jong: Insular Southeast Asian Fleets in Trade and War (Fifteenth to Seventeenth Centuries)", in A. Reid (éd.), Southeast Asia in the Early Modern Era, Ithaca/London, Cornell Univ. Press, 1993: 197-213.

_, "Demografi dan tata perkotaan di Aceh pada abad ke-16: Data baru menurut sebuah buku pedoman Portugis tahun 1584", in H. Chambert-Loir \& H. Muarif Ambary (éds.), Panggung Sejarah. Persembahan kepada Prof. Dr. Denys Lombard, Jakarta, EFEO, Pusat Penelitian dan Pengembangan Arkeologi Nasional, Yayasan Pustaka Obor Indonesia, 2011: 225-244 (1 êre éd. 1999).

Meilink-Roelofsz, M.A.P., Asian Trade and European Influence in the Indonesian Archipelago between 1500 and about 1630, The Hague, M. Nijhoff, 1962.

Pathmanathan, S., "The Lankatilaka Temple Inscriptions of a Merchant Community", in N. Karashima (éd.), Ancient and Medieval Commercial Activities in the Indian Ocean: Testimony of Inscriptions and Ceramic-sherds, Taisho Univ., Report on the Taisho Univ. Research Project, 1997-2000, 2002: 36-47.

Perret, Daniel, «Barus : société et relations extérieures (XII'-milieu du XVII ${ }^{\mathrm{e}} \mathrm{s}$.)», in D. Perret \& H. Surachman (ed.), Histoire de Barus-Sumatra. III : Regards sur une place marchande de l'océan Indien (XII ${ }^{e}$-milieu du XVII ${ }^{e}$ s.), Paris, EFEO/Archipel (cahier d'Archipel 38), 2009 : 533-642.

-, "From Slave to King: The Role of South Asians in Maritime Southeast Asia (from the late $13^{\text {th }}$ to the late $17^{\text {th }}$ century)", Archipel, 82: 159-199.

Perret, Daniel; Kamarudin Ab. Razak \& Kalus, Ludvik, Batu Aceh. Warisan Sejarah Johor, Johor Bahru, École française d'Extrême-Orient/Yayasan Warisan Johor, 1999.

Perret, D.; Surachman, H.; Péronnet, S. ; Hidayat, D.; Soedewo, E. ; Susilowati, N. ; Utomo, R.W.; Sutrisna, D.; Sunaryo, U., "Poteries», in D. Perret \& H. Surachman (ed.), Histoire de Barus-Sumatra. III : Regards sur une place marchande de l'océan Indien (XII ${ }^{e}$-milieu du XVII s.), Paris, EFEO/Archipel (cahier d'Archipel 38), 2009 : 153-300.

Pradham, Debaraj; Mohanty, Pradeep \& Misra, Jitu, "Manikapatana: an ancient and medieval 
port on the coast of Orissa”, in Kishor K. Basa \& Pradeep Mohanty (éd.), Archaeology of Orissa, Delhi, Pratibha Prakashan, 2000, vol. II : 473-494.

Prakash, Om, "The European Trading Companies and the Merchants of Bengal, 1650-1725", IESHR, 1(1), 1963: 37-63.

- "The Mughal empire and the Dutch East Indies Company in the seventeenth century", in E. Locher-Scholten \& P. Rietbergen (éd.), Hof en Handel. Aziatische vorsten en de VOC 1620-1720, Leiden, KITLV Uitgeverij, 2004a: 183-200.

_, “The Indian Maritime Merchant, 1500-1800”, JESHO, 47(3), 2004b: 435-457.

Ptak, Roderich, "Yuan and Early Ming Notices on the Kayal Area in South India", BEFEO, 80(1), 1993: 137-156.

Rao, K.P., "Kottapatnam-A South Indian Port Trading with Eastern Lands", in N. Karashima (éd.), Ancient and Medieval Commercial Activities in the Indian Ocean: Testimony of Inscriptions and Ceramic-sherds, Taisho Univ., Report on the Taisho Univ. Research Project, 1997-2000, 2002: 125-133.

Ray, Haraprasad, "The South East Asian Connection in Sino-Indian Trade", in R. Scott \& J. Guy (éd.), South East Asia \& China: Art, Interaction \& Commerce, London, Percival David Foundation of Chinese Art, SOAS, 1995: 41-54.

Raychaudhuri, Tapan, Jan Company in Coromandel (1605-1690), 's-Gravenhage, M. Nijhoff, VKI 38, 1962.

Reid, Anthony, Southeast Asia in the Age of Commerce, 1450-1680, New Haven and London, Yale University Press, 1988-1993, 2 vol.

Rokus Due Awe, «Faune», in D. Perret \& H. Surachman (ed.), Histoire de Barus-Sumatra. III : Regards sur une place marchande de l'océan Indien (XIIe-milieu du XVII $s$.$) , Paris,$ EFEO/Archipel (cahier d'Archipel 38), 2009 : 455-462.

Salmon, Claudine, «La sériciculture à Sumatra Nord. De 1'histoire à la légende de Job», Archipel, 70, 2005 : 239-260.

Sasaki, H., "Chinese and Thai Ceramics in Kottapatnam”, in N. Karashima (éd.), Ancient and Medieval Commercial Activities in the Indian Ocean: Testimony of Inscriptions and Ceramic-sherds, Taisho Univ., Report on the Taisho Univ. Research Project, 1997-2000, 2002: 134-144.

Schrieke, B., Indonesian sociological studies, vol. II, The Hague/Bandung, van Hoeve, 1957.

Sen, S.P., "The Role of Indian Textiles in Southeast Asian Trade in the Seventeenth Century", JSEAH, 3(2), 1962: 92-110.

Sridhar, T.S. (éd.), Excavations of Archaeological sites in Tamilnadu [1969-1995], Chennai, Tamil Nadu State Department of Archaeology, 2004.

Stephen, S. Jeyaseela, Portuguese in the Tamil Coast, Pondicherry, Navajothi, 1998.

Subbarayalu, Y., "Chinese Ceramics of Tamilnadu and Kerala Coasts", in H.P. Ray \& J.-F. Salles (éd.), Tradition and Archaeology. Early Maritime Contacts in the Indian Ocean, New Delhi, nistads, Lyon, Maison de l'Orient Méditerranéen, 1996: 109-114.

-, "Anjuvannan: A Maritime Trade Guild of Medieval Times", in H. Kulke, K. Kesavapany, V. Sakhuja (eds.), Nagapattinam to Suvarnadwipa: Reflections on the Chola Naval Expeditions to Southeast Asia, Singapore, ISEAS, 2009: 158-167.

-, "A trade guild Tamil inscription at Neusu, Aceh", in D. Perret \& H. Surachman (eds.), Histoire de Barus. III : Regards sur une place marchande de l'océan Indien (XIIe-milieu $d u$ XVII s. ), Paris, EFEO/Cahier d'Archipel 38, 2009a : 529-532.

Subrahmanyam, Sanjay, Improvising Empire. Portuguese Trade and Settlement in the Bay of Bengal, 1500-1700, Delhi, Oxford University Press, 1990a.

- The Political Economy of Commerce: Southern India 1500-1650, Cambridge, Cambridge University Press, 1990b.

—, "The Coromandel Malacca Trade in the Sixteenth Century: A Study of its Evolving", in 
Om Prakash (éd.), European Commercial Expansion in Early Modern Asia, Aldershot, Variorum, 1997: 25-42.

-, “'Persianization' and 'Mercantilism': Two Themes in Bay of Bengal History, 14001700", in Om Prakash \& D. Lombard (ed.), Commerce and Culture in the Bay of Bengal, 1500-1800, Delhi, Manohar/Indian Council of Historical Research, 1999: 47-85.

Thomaz, Luis Filipe F.R., "The Malay Sultanate of Melaka", in A. Reid (ed.), Southeast Asia in the Early Modern Era, Ithaca/London, Cornell University Press, 1993: 69-90.

Tripati, Sila \& Vora, K.H., "Maritime heritage in and around Chilika Lake, Orissa: Geological evidences for its decline", Current Science, 88(7), 10/04/2005: 1175-1181.

Winks, André, Al-Hind. The Making of the Indo-Islamic World. Vol. I: Early Medieval India and the Expansion of Islam (7th-11 $1^{\text {th }}$ centuries), Leiden, E.J. Brill, 1996. 